\title{
CLAY MINERAL CONTENT OF CONTINENTAL SHELF AND RIVER SEDIMENTS, SOUTHERN CALIFORNIA
}

By

James R. Hein ${ }^{1}$ and Jennifer S. Dowling ${ }^{1}$

Open File Report 01-77

2001

This report is preliminary and has not been reviewed for conformity with U.S. Geological Survey editorial standards or with the North American Stratigraphic Code. Any use of trade, firm, or product names is for descriptive purposes only and does not imply endorsement by the U.S. Government.

\section{U.S. DEPARTMENT OF THE INTERIOR}

U.S. GEOLOGICAL SURVEY

${ }^{\mathrm{T}}$ U.S. Geological Survey, 345 Middlefield Rd., MS999, Menlo Park, CA, 94025 


\section{EXPLANATION}

This report contains data on the clay mineral content of 250 shelf surface-sediment samples from the California Continental Borderland (Tables 1, 2; Figures 1-7), 79 samples with depth in cores from Santa Monica Bay (Table 3; see Table 1 for surface sediment data for those same cores and for core locations), 24 suspended and 13 bottom sediment samples from rivers draining Southern California (Table 4), and six rock samples or composite rock samples from the Palos Verdes Headland (Table 4). This report is designed as the data repository and these data are discussed in a paper by Hein et al. (2001). Summary tables of the data presented here and table 4 presented here are included in that paper. The techniques used in sample preparation and analyses are repeated here.

\section{METHOD OF CLAY MINERAL ANALYSIS}

The procedure used to determine clay mineral suites includes the following steps of sample treatment. 1. About 5-15 cc of sediment were soaked for 2 days in Morgan's solution (sodium acetate, glacial acetic acid, and water) to remove carbonate; and $30 \%$ hydrogen peroxide to remove organic matter, after which the samples were washed several times with Morgan's solution and then removed from suspension by centrifugation. 2. The washed sediment was suspended again with a magnetic stirrer in $0.01 \%$ sodium carbonate solution and the $<2 \mu \mathrm{m}$ size fraction was isolated by centrifugation. 3 . The clay-sized material was concentrated by centrifuging; magnesium saturated by washing 4 times with $1 \mathrm{M}$ magnesium chloride; and excess salts were removed by washing with distilled water. 4 . The clay was spread evenly over a plastic or glass insert of an aluminum sample holder and dried in a desiccater, after which the sample was placed over a bowl of ethylene glycol in an oven at $65^{\circ} \mathrm{C}$ for 1 hour and then in a desiccater for 1 day to expand the smectite clay mineral lattice so that smectite could be distinguished from chlorite and vermiculite.

The glycolated samples were analyzed using a Philips X-ray diffractometer with carbon monochromator and Cuk $\alpha$ radiation. Continuous scans were run from $3-70^{\circ} 2 \Theta$ using a step size of $0.02^{\circ} 2 \Theta$ at $1 \mathrm{~s} / \mathrm{step}$, and scan speed of $0.02^{\circ} 2 \Theta / \mathrm{s}$. A second scan was run from $24-26^{\circ} 2 \Theta$ using a step size of $0.02^{\circ} 2 \Theta$ at $5 \mathrm{~s} / \mathrm{step}$, and scan speed of $0.004^{\circ} 2 \Theta / \mathrm{s}$. This second scan was used to attempt to distinguish chlorite from kaolinite (Biscaye, 1964), which was not routinely possible. So, kaolinite and chlorite percentages are listed together. Semi-quantitative estimates of relative clay mineral percentages were obtained from measurement of peak-areas, which were multiplied times the weighting factors of Biscaye (1965), which are 4 times the illite peak area, 2 times the kaolinite + chlorite peak area, and 1 times the smectite peak area, then summed to $100 \%$. This procedure allows us to compare our results with many other studies of marine clay minerals that use those same weighting factors. In addition, the un-weighted clay mineral contents are listed in table 2. Smectite is a smectite/illite mixed layer clay mineral and the amount of expandable layers (the smectite portion) was also calculated based on the difference in the height of the base line from the high and low $2 \Theta$ sides of the $5.2^{\circ} 2 \Theta$ peak (see Perry and Hower, 1970; and Reynolds and Hower, 1970). 
In order to measure analytical precision, duplicate analyses were run on $10 \%$ of the samples (Table 5). Reproducibility was within 3 percentage points for $80 \%$ of the duplicate samples and within 5 points for $90 \%$ of the samples. Samples with total peak areas less than about $550-650 \mathrm{~mm}^{2}$ generally had poor reproducibility and those data are not used for surface sediment samples, but are used for some core samples when the clay mineral contents calculated from those diffractograms are comparable with clay mineral contents from either underlying or overlying samples; these samples are listed in the tables as LCMC, low clay mineral content. The potentially useable LCMC data listed in table 3 from the cores are flagged by parentheses around the clay mineral contents. A less than $2 \mu \mathrm{m}$ fraction exists for those LCMC samples, but the clay minerals are either absent or very poorly crystalline. Accuracy depends on the applicability of the weighting factors to the clay minerals analyzed in this study, and that cannot be evaluated.

\section{REFERENCE CITED}

Biscaye, P.E., 1964. Distinction between kaolinite and chlorite in recent sediment by Xray diffraction. American Mineralogist 49, 1281-2189.

Biscaye, P.E., 1965. Mineralogy and sedimentation of recent deep sea clay in the Atlantic Ocean and adjacent seas and oceans. Geological Society of America Bulletin 76, 803-832.

Griggs, G. B. and Hein, J. R., 1980. Sources, dispersal, and clay mineral composition of fine-grained sediment off central and northern California. Journal Geology 88, 541566.

Hein, J.R., Dowling, J.S., Schuetze, A., and Lee, H.J., 2001. Clay mineral suites, sources, and inferred dispersal routes: California Continental Borderland shelf. Marine Environmental Research (submitted).

Perry, E. and Hower, J., 1970. Burial diagenesis in Gulf Coast pelitic sediments. Clays and Clay Minerals 18, 165-177.

Reynolds, R.C., Jr. and Hower, J., 1970. The nature of interlayering in mixed-layer illitemontmorillonites. Clays and Clay Minerals 18, 25-36.

Key Search Words: Clay mineralogy, illite, smectite, kaolinite, chlorite, expandable layers, Southern California Borderland, continental shelf, shelf sediments, fine-grained sediment, clay-size fraction, river sediments 
Table 1. Weighted* clay mineral content of the $<2 \mu \mathrm{m}$ size-fraction of Southern California Borderland shelf surface sediments (locations in Fig. 1)

\begin{tabular}{|c|c|c|c|c|c|c|c|}
\hline Map \# & $\begin{array}{l}\text { Sample } \\
\text { Identification }\end{array}$ & $\begin{array}{c}\text { Smectite } \\
(\%)\end{array}$ & $\begin{array}{l}\text { Illite } \\
(\%)\end{array}$ & $\begin{array}{c}\mathrm{K}+\mathrm{C} \\
(\%)\end{array}$ & $\begin{array}{c}\text { Expandable } \\
\text { Layers } \\
(\%)\end{array}$ & $\begin{array}{c}\text { Latitude } \\
\left({ }^{\circ} \mathrm{N}\right)\end{array}$ & $\begin{array}{l}\text { Longitude } \\
\left({ }^{\circ} \mathrm{W}\right)\end{array}$ \\
\hline 1 & 2354 & 31 & 43 & 26 & 50 & 34.45885 & 120.31283 \\
\hline 2 & 2370 & 39 & 41 & 20 & 56 & 34.29863 & 119.36180 \\
\hline 3 & 2338 & 26 & 44 & 30 & 50 & 34.26987 & 119.34127 \\
\hline 4 & 2340 & 24 & 47 & 29 & 49 & 34.26512 & 119.34163 \\
\hline 5 & 2339 & 22 & 48 & 30 & 55 & 34.25232 & 119.28482 \\
\hline 6 & 2331 & 40 & 37 & 23 & 60 & 34.24563 & 119.28873 \\
\hline 7 & 2329 & LCMC & LCMC & LCMC & LCMC & 34.25008 & 119.27637 \\
\hline 8 & 2330 & 0 & 54 & 46 & 0 & 34.23993 & 119.28082 \\
\hline 9 & 2328 & 0 & 57 & 43 & 0 & 34.21907 & 119.28765 \\
\hline 10 & 2375 & 23 & 49 & 28 & 49 & 34.18795 & 119.35537 \\
\hline 11 & 2376 & 21 & 48 & 31 & 52 & 34.17882 & 119.34637 \\
\hline 12 & 2129 & 14 & 59 & 27 & 75 & 34.17317 & 119.22782 \\
\hline 13 & 2130 & 0 & 55 & 45 & 0 & 34.17183 & 119.22330 \\
\hline 14 & 2131 & 32 & 47 & 21 & 62 & 34.16277 & 119.22467 \\
\hline 15 & 2132 & 0 & 80 & 20 & 0 & 34.15490 & 119.22723 \\
\hline 16 & 2273 & 6 & 49 & 45 & 40 & 34.13045 & 119.19843 \\
\hline 17 & 2275 & 27 & 53 & 20 & 41 & 34.13072 & 119.19548 \\
\hline 18 & 2274 & 49 & 28 & 23 & 65 & 34.12380 & 119.19745 \\
\hline 19 & 2276 & 11 & 72 & 17 & 48 & 34.12367 & 119.18282 \\
\hline 20 & 2341 & 31 & 43 & 26 & 58 & 34.08998 & 119.15002 \\
\hline 21 & 2342 & 23 & 45 & 32 & 59 & 34.07048 & 119.15975 \\
\hline 22 & 2310 & 52 & 32 & 16 & 64 & 34.09157 & 119.08910 \\
\hline 23 & 2308 & 31 & 48 & 21 & 60 & 34.08305 & 119.09022 \\
\hline 24 & 2377 & LCMC & LCMC & LCMC & LCMC & 34.03890 & 118.94028 \\
\hline 25 & 2378 & LCMC & LCMC & LCMC & LCMC & 34.03253 & 118.92253 \\
\hline 26 & 2343 & 20 & 66 & 14 & 47 & 34.02463 & 118.85227 \\
\hline 27 & 2344 & 52 & 30 & 18 & 70 & 34.01922 & 118.85678 \\
\hline 28 & 2379 & 40 & 34 & 26 & 55 & 34.01922 & 118.83677 \\
\hline 29 & S2-97-SC 26B1/0-2 & 46 & 34 & 20 & 70 & 33.99433 & 118.77200 \\
\hline 30 & 2381 & 41 & 36 & 23 & 67 & 34.00250 & 118.76933 \\
\hline 31 & 2380 & 52 & 32 & 16 & 62 & 34.01825 & 118.76010 \\
\hline 32 & S2-97-SC 54B1/0-2 & 53 & 25 & 22 & 56 & 34.02267 & 118.65900 \\
\hline 33 & S2-97-SC $6 \mathrm{~B} 1 / 0-2$ & 41 & 39 & 20 & 62 & 34.02117 & 118.65417 \\
\hline 34 & 2382 & 54 & 30 & 16 & 70 & 34.02307 & 118.59257 \\
\hline 35 & S2-97-SC 4B1/0-2 & 19 & 53 & 28 & 56 & 34.01350 & 118.53405 \\
\hline 36 & S2-97-SC $24 \mathrm{~B} 1 / 0-2$ & 39 & 39 & 22 & 63 & 33.97117 & 118.65150 \\
\hline 37 & S2-97-SC 18B1/0-2 & 33 & 41 & 26 & 61 & 33.97933 & 118.57517 \\
\hline 38 & S2-97-SC $22 \mathrm{~B} 1 / 0-2$ & 39 & 39 & 22 & 62 & 33.98017 & 118.55683 \\
\hline 39 & S2-97-SC 20B1/0-2 & 41 & 34 & 25 & 58 & 33.96883 & 118.53217 \\
\hline 40 & 2305 & 24 & 61 & 15 & 47 & 33.97573 & 118.47113 \\
\hline 41 & 2307 & LCMC & LCMC & LCMC & LCMC & 33.96225 & 118.47670 \\
\hline 42 & S2-97-SC 16B1/0-2 & 23 & 49 & 28 & 50 & 33.94183 & 118.58983 \\
\hline 43 & S2-97-SC 51B1/0-2 & 21 & 47 & 32 & 56 & 33.94283 & 118.57483 \\
\hline 44 & 2192 & 44 & 31 & 25 & 51 & 33.94450 & 118.51908 \\
\hline 45 & S2-97-SC 49B1/0-2 & 22 & 59 & 19 & 42 & 33.95033 & 118.48233 \\
\hline 46 & S2-97-SC 53B3/0-2 & LCMC & LCMC & LCMC & LCMC & 33.92833 & 118.55433 \\
\hline 47 & S2-97-SC 10B1/0-2 & 38 & 41 & 21 & 53 & 33.92133 & 118.56017 \\
\hline 48 & S2-97-SC 52B1/0-2 & 40 & 31 & 29 & 47 & 33.91383 & 118.57233 \\
\hline 49 & S2-97-SC 50B1/0-2 & 41 & 35 & 24 & 64 & 33.92783 & 118.53500 \\
\hline 50 & S2-97-SC 40B1/0-2 & 42 & 37 & 21 & 65 & 33.92517 & 118.53450 \\
\hline 51 & S2-97-SC 42B1/0-2 & 34 & 46 & 20 & 64 & 33.93333 & 118.50667 \\
\hline 52 & 2306 & 0 & 87 & 13 & 0 & 33.93588 & 118.45358 \\
\hline 53 & 2384 & 30 & 44 & 26 & 58 & 33.92987 & 118.50390 \\
\hline
\end{tabular}


Table 1 continued

\begin{tabular}{|c|c|c|c|c|c|c|c|}
\hline Map \# & $\begin{array}{l}\text { Sample } \\
\text { Identification }\end{array}$ & $\begin{array}{c}\text { Smectite } \\
(\%)\end{array}$ & $\begin{array}{l}\text { Illite } \\
(\%)\end{array}$ & $\begin{array}{c}\mathrm{K}+\mathrm{C} \\
(\%)\end{array}$ & $\begin{array}{c}\text { Expandable } \\
\text { Layers } \\
(\%)\end{array}$ & $\begin{array}{l}\text { Latitude } \\
\left({ }^{\circ} \mathrm{N}\right)\end{array}$ & $\begin{array}{c}\text { Longitude } \\
\left({ }^{\circ} \mathrm{W}\right)\end{array}$ \\
\hline 54 & S2-97-SC 44B1/0-2 & 22 & 56 & 22 & 50 & 33.92483 & 118.50050 \\
\hline 55 & 2195 & 52 & 31 & 17 & 65 & 33.91088 & 118.52502 \\
\hline 56 & S2-97-SC 55B1/0-2 & 34 & 40 & 26 & 43 & 33.90900 & 118.52517 \\
\hline 57 & 2197 & 44 & 36 & 20 & 61 & 33.90387 & 118.50077 \\
\hline 58 & S2-97-SC 34B2/0-2 & 38 & 37 & 25 & 65 & 33.88667 & 118.47800 \\
\hline 59 & 2386 & 4 & 88 & 8 & 41 & 33.87808 & 118.42445 \\
\hline 60 & S2-97-SC 28B1/0-2 & 41 & 40 & 19 & 52 & 33.83850 & 118.55133 \\
\hline 61 & S2-97-SC 36B1/0-2 & 26 & 49 & 25 & 61 & 33.83333 & 118.51133 \\
\hline 62 & S2-97-SC 33B $1 / 0-2$ & 34 & 42 & 24 & 59 & 33.84717 & 118.46100 \\
\hline 63 & 2387 & 30 & 45 & 25 & 61 & 33.84833 & 118.45215 \\
\hline 64 & S2-97-SC 48B1/0-2 & 31 & 43 & 26 & 55 & 33.83850 & 118.42700 \\
\hline 65 & 2200 & 37 & 40 & 23 & 58 & 33.74790 & 118.42902 \\
\hline 66 & 2201 & 35 & 44 & 21 & 56 & 33.72067 & 118.39237 \\
\hline 67 & 2202 & 46 & 34 & 20 & 60 & 33.72207 & 118.37248 \\
\hline 68 & 2389 & LCMC & LCMC & LCMC & LCMC & 33.70948 & 118.32180 \\
\hline 69 & 2204 & 32 & 45 & 23 & 54 & 33.69690 & 118.33898 \\
\hline 70 & 2205 & 27 & 48 & 25 & 57 & 33.67685 & 118.30577 \\
\hline 71 & 2206 & 32 & 44 & 24 & 65 & 33.67510 & 118.29328 \\
\hline 72 & 2390 & 17 & 48 & 35 & 60 & 33.69353 & 118.26472 \\
\hline 73 & 2394 & 34 & 47 & 19 & 61 & 33.65108 & 118.24908 \\
\hline 74 & 2392 & 12 & 69 & 19 & 42 & 33.70513 & 118.15537 \\
\hline 75 & 2391 & 21 & 54 & 25 & 52 & 33.70947 & 118.13780 \\
\hline 76 & 2393 & 0 & 78 & 22 & 0 & 33.68292 & 118.08832 \\
\hline 77 & 2395 & 6 & 72 & 22 & 48 & 33.67270 & 118.05412 \\
\hline 78 & 2396 & 15 & 53 & 32 & 65 & 33.64805 & 118.14943 \\
\hline 79 & 2397 & 29 & 45 & 26 & 56 & 33.65007 & 118.12595 \\
\hline 80 & 2398 & 31 & 35 & 34 & 60 & 33.61928 & 118.14298 \\
\hline 81 & 2400 & 22 & 52 & 26 & 61 & 33.60333 & 118.09537 \\
\hline 82 & 2207 & 23 & 49 & 28 & 70 & 33.61262 & 118.06588 \\
\hline 83 & 2208 & 34 & 40 & 26 & 58 & 33.60222 & 118.05647 \\
\hline 84 & 2209 & 36 & 44 & 20 & 65 & 33.59203 & 118.06285 \\
\hline 85 & 2210 & 29 & 45 & 26 & 45 & 33.59022 & 118.04122 \\
\hline 86 & 2399 & 25 & 48 & 27 & 50 & 33.63473 & 117.99482 \\
\hline 87 & 2325 & 10 & 68 & 22 & 42 & 33.62767 & 117.98723 \\
\hline 88 & 2453 & 0 & 59 & 41 & 0 & 33.62787 & 117.97487 \\
\hline 89 & 2326 & 7 & 76 & 17 & 47 & 33.62273 & 117.95618 \\
\hline 90 & 2211 & 24 & 50 & 26 & 58 & 33.58802 & 118.01003 \\
\hline 91 & 2212 & 28 & 48 & 24 & 57 & 33.59005 & 117.98927 \\
\hline 92 & 2213 & 30 & 43 & 27 & 50 & 33.57152 & 117.97777 \\
\hline 93 & 2401 & 26 & 51 & 23 & 65 & 33.59168 & 117.95748 \\
\hline 94 & 2402 & 34 & 52 & 14 & 65 & 33.55740 & 117.83023 \\
\hline 95 & 2403 & 36 & 47 & 17 & 70 & 33.51870 & 117.80285 \\
\hline 96 & 2303 & 15 & 55 & 30 & 40 & 33.51870 & 117.78187 \\
\hline 97 & 2304 & LCMC & LCMC & LCMC & LCMC & 33.52132 & 117.76962 \\
\hline 98 & 2279 & 45 & 35 & 20 & 70 & 33.51542 & 117.76638 \\
\hline 99 & 2280 & 31 & 49 & 20 & 70 & 33.50842 & 117.76908 \\
\hline 100 & 2302 & 34 & 47 & 19 & 70 & 33.50998 & 117.77635 \\
\hline 101 & 2345 & 34 & 45 & 21 & 73 & 33.50668 & 117.76847 \\
\hline 102 & 2346 & 38 & 43 & 19 & 75 & 33.49363 & 117.77703 \\
\hline 103 & 2405 & 31 & 45 & 24 & 55 & 33.29462 & 117.55857 \\
\hline 104 & 2406 & 26 & 51 & 23 & 53 & 33.28140 & 117.47188 \\
\hline 105 & 2407 & 19 & 50 & 31 & 53 & 33.26032 & 117.52368 \\
\hline 106 & 2286 & 26 & 48 & 26 & 65 & 33.16665 & 117.38845 \\
\hline
\end{tabular}


Table 1 continued

\begin{tabular}{|c|c|c|c|c|c|c|c|}
\hline Map \# & $\begin{array}{l}\text { Sample } \\
\text { Identification }\end{array}$ & $\begin{array}{l}\text { Smectite } \\
(\%)\end{array}$ & $\begin{array}{l}\text { Illite } \\
(\%)\end{array}$ & $\begin{array}{l}\mathrm{K}+\mathrm{C} \\
(\%)\end{array}$ & $\begin{array}{c}\text { Expandable } \\
\text { Layers } \\
(\%)\end{array}$ & $\begin{array}{c}\text { Latitude } \\
\left({ }^{\circ} \mathrm{N}\right)\end{array}$ & $\begin{array}{l}\text { Longitude } \\
\left({ }^{\circ} \mathrm{W}\right)\end{array}$ \\
\hline 107 & 2285 & 15 & 52 & 33 & 61 & 33.15912 & 117.39795 \\
\hline 108 & 2288 & 22 & 51 & 27 & 65 & 33.15862 & 117.38435 \\
\hline 109 & 2287 & 20 & 55 & 25 & 62 & 33.15913 & 117.38960 \\
\hline 110 & 2292 & 26 & 52 & 22 & 59 & 33.11928 & 117.35648 \\
\hline 111 & 2290 & 19 & 56 & 25 & 67 & 33.11623 & 117.35880 \\
\hline 112 & 2455 & 13 & 70 & 17 & 51 & 33.10973 & 117.34688 \\
\hline 113 & 2408 & 21 & 59 & 20 & 67 & 33.10512 & 117.36183 \\
\hline 114 & 2289 & 22 & 52 & 26 & 61 & 33.10245 & 117.35848 \\
\hline 115 & 2291 & 28 & 50 & 22 & 65 & 33.09985 & 117.35260 \\
\hline 116 & 2456 & 20 & 54 & 26 & 47 & 33.01013 & 117.30225 \\
\hline 117 & 2294 & 21 & 53 & 26 & 57 & 33.00282 & 117.30477 \\
\hline 118 & 2409 & 5 & 82 & 13 & 48 & 33.00405 & 117.28200 \\
\hline 119 & 2293 & LCMC & LCMC & LCMC & LCMC & 32.99953 & 117.29225 \\
\hline 120 & 2296 & 19 & 53 & 28 & 53 & 32.99895 & 117.29713 \\
\hline 121 & 2295 & 19 & 51 & 30 & 60 & 32.99040 & 117.29865 \\
\hline 122 & 2349 & 31 & 40 & 29 & 65 & 32.89455 & 117.26883 \\
\hline 123 & 2350 & 17 & 51 & 32 & 70 & 32.88863 & 117.27770 \\
\hline 124 & 2351 & 16 & 61 & 23 & 56 & 32.82150 & 117.32023 \\
\hline 125 & 2410 & LCMC & LCMC & LCMC & LCMC & 32.77215 & 117.35455 \\
\hline 126 & 2411 & 24 & 54 & 22 & 61 & 32.75255 & 117.34298 \\
\hline 127 & 2412 & 12 & 75 & 13 & 41 & 32.72142 & 117.29627 \\
\hline 128 & 2215 & 23 & 57 & 20 & 65 & 32.68123 & 117.31000 \\
\hline 129 & 2216 & 19 & 54 & 27 & 50 & 32.67618 & 117.32203 \\
\hline 130 & 2219 & 25 & 53 & 22 & 61 & 32.65480 & 117.33142 \\
\hline 131 & 2220 & 15 & 58 & 27 & 55 & 32.65968 & 117.28002 \\
\hline 132 & 2414 & 5 & 81 & 14 & 40 & 32.67698 & 117.18978 \\
\hline 133 & 2415 & 6 & 76 & 18 & 50 & 32.65873 & 117.18228 \\
\hline 134 & 2417 & 2 & 89 & 9 & 47 & 32.62497 & 117.21460 \\
\hline 135 & 2418 & 20 & 65 & 15 & 50 & 32.59530 & 117.31480 \\
\hline 136 & 2419 & 8 & 85 & 7 & 41 & 32.58938 & 117.26360 \\
\hline 137 & 2353 & 15 & 65 & 20 & 49 & 32.55213 & 117.26375 \\
\hline 138 & 2352 & 24 & 58 & 18 & 54 & 32.54187 & 117.19028 \\
\hline 139 & 2335 & 10 & 76 & 14 & 41 & 32.54470 & 117.15480 \\
\hline 140 & 2169 & 21 & 54 & 25 & 60 & 33.76823 & 118.27835 \\
\hline 141 & 2174 & 16 & 54 & 30 & 58 & 33.73425 & 118.26655 \\
\hline 142 & 2178 & 21 & 53 & 26 & 55 & 33.72800 & 118.27103 \\
\hline 143 & 2184 & 21 & 54 & 25 & 50 & 33.72060 & 118.26907 \\
\hline 144 & 2170 & 18 & 59 & 23 & 53 & 33.76432 & 118.25600 \\
\hline 145 & 2176 & 26 & 39 & 35 & 60 & 33.73098 & 118.26112 \\
\hline 146 & 2182 & 22 & 45 & 33 & 60 & 33.72382 & 118.26233 \\
\hline 147 & 2428 & 23 & 48 & 29 & 58 & 33.71883 & 118.25853 \\
\hline 148 & 2168 & 28 & 48 & 24 & 63 & 33.71192 & 118.25063 \\
\hline 149 & 2421 & 23 & 48 & 29 & 61 & 33.76625 & 118.24090 \\
\hline 150 & 2172 & 15 & 53 & 32 & 61 & 33.74922 & 118.24268 \\
\hline 151 & 2177 & 14 & 46 & 40 & 45 & 33.73478 & 118.24262 \\
\hline 152 & 2162 & 24 & 46 & 30 & 63 & 33.71345 & 118.24173 \\
\hline 153 & 2173 & 20 & 49 & 31 & 60 & 33.74727 & 118.23767 \\
\hline 154 & 2300 & 25 & 48 & 27 & 60 & 33.71802 & 118.23905 \\
\hline 155 & 2299 & 31 & 44 & 25 & 64 & 33.72058 & 118.23400 \\
\hline 156 & 2297 & 24 & 52 & 24 & 60 & 33.72285 & 118.23532 \\
\hline 157 & 2298 & 23 & 48 & 29 & 56 & 33.72892 & 118.23398 \\
\hline 158 & 2427 & 23 & 48 & 29 & 58 & 33.73087 & 118.23550 \\
\hline 159 & 2426 & 28 & 49 & 23 & 63 & 33.73415 & 118.23143 \\
\hline
\end{tabular}


Table 1 continued

\begin{tabular}{|c|c|c|c|c|c|c|c|}
\hline Map \# & $\begin{array}{l}\text { Sample } \\
\text { Identification }\end{array}$ & $\begin{array}{c}\text { Smectite } \\
(\%)\end{array}$ & $\begin{array}{l}\text { Illite } \\
(\%)\end{array}$ & $\begin{array}{c}\mathrm{K}+\mathrm{C} \\
(\%)\end{array}$ & $\begin{array}{c}\text { Expandable } \\
\text { Layers } \\
(\%)\end{array}$ & $\begin{array}{l}\text { Latitude } \\
\left({ }^{\circ} \mathrm{N}\right)\end{array}$ & $\begin{array}{c}\text { Longitude } \\
\left({ }^{\circ} \mathrm{W}\right)\end{array}$ \\
\hline 160 & 2175 & 23 & 50 & 27 & 61 & 33.74087 & 118.22667 \\
\hline 161 & 2432 & 18 & 56 & 26 & 56 & 33.75080 & 118.23037 \\
\hline 162 & 2430 & 25 & 51 & 24 & 57 & 33.76910 & 118.22448 \\
\hline 163 & 2431 & 23 & 51 & 26 & 57 & 33.75337 & 118.22408 \\
\hline 164 & 2179 & 14 & 58 & 28 & 55 & 33.73895 & 118.21028 \\
\hline 165 & 2158 & 23 & 51 & 26 & 59 & 33.72830 & 118.20863 \\
\hline 166 & 2159 & 26 & 50 & 24 & 63 & 33.72253 & 118.21038 \\
\hline 167 & 2450 & 39 & 50 & 11 & 53 & 33.76025 & 118.19937 \\
\hline 168 & 2185 & 15 & 58 & 27 & 58 & 33.73318 & 118.19975 \\
\hline 169 & 2160 & 20 & 54 & 26 & 54 & 33.72357 & 118.20468 \\
\hline 170 & 2161 & 27 & 51 & 22 & 65 & 33.72352 & 118.20167 \\
\hline 171 & 2186 & 21 & 51 & 28 & 59 & 33.73137 & 118.19300 \\
\hline 172 & 2311 & 43 & 39 & 18 & 62 & 33.75550 & 118.18500 \\
\hline 173 & 2187 & 29 & 48 & 23 & 60 & 33.73118 & 118.18402 \\
\hline 174 & 2451 & 39 & 45 & 16 & 57 & 33.75188 & 118.17423 \\
\hline 175 & 2155 & 19 & 60 & 21 & 55 & 33.74330 & 118.16753 \\
\hline 176 & 2156 & 34 & 53 & 13 & 63 & 33.73997 & 118.17133 \\
\hline 177 & 2163 & 20 & 54 & 26 & 58 & 33.72793 & 118.16683 \\
\hline 178 & 2152 & 18 & 60 & 22 & 50 & 33.75932 & 118.16267 \\
\hline 179 & 2153 & 25 & 51 & 24 & 61 & 33.75350 & 118.15783 \\
\hline 180 & 2154 & 26 & 54 & 20 & 60 & 33.74902 & 118.15887 \\
\hline 181 & 2388 & 31 & 50 & 19 & 63 & 33.74833 & 118.14870 \\
\hline 182 & 2157 & 17 & 58 & 25 & 50 & 33.74232 & 118.15318 \\
\hline 183 & 2167 & 30 & 49 & 21 & 63 & 33.73557 & 118.15765 \\
\hline 184 & 2319 & 30 & 52 & 18 & 62 & 33.73712 & 118.14350 \\
\hline 185 & 2320 & 14 & 66 & 20 & 46 & 33.73297 & 118.12148 \\
\hline 186 & 2321 & 7 & 60 & 33 & 51 & 33.72932 & 118.13347 \\
\hline 187 & 2318 & 33 & 48 & 19 & 65 & 33.72370 & 118.12690 \\
\hline 188 & 2188 & 26 & 54 & 20 & 61 & 33.73383 & 118.08880 \\
\hline 189 & 2164 & 25 & 53 & 22 & 62 & 33.73033 & 118.08262 \\
\hline 190 & 2134 & 25 & 55 & 20 & 50 & 33.71543 & 118.06218 \\
\hline 191 & 2136 & 37 & 53 & 10 & 64 & 33.61888 & 117.92720 \\
\hline 192 & 2137 & 41 & 48 & 11 & 65 & 33.61300 & 117.92387 \\
\hline 193 & 2138 & 36 & 53 & 11 & 67 & 33.61408 & 117.91412 \\
\hline 194 & 2142 & 48 & 43 & 9 & 70 & 33.60773 & 117.91003 \\
\hline 195 & 2143 & 52 & 40 & 8 & 75 & 33.60697 & 117.90633 \\
\hline 196 & 2141 & 42 & 49 & 9 & 75 & 33.61135 & 117.90223 \\
\hline 197 & 2144 & 43 & 49 & 8 & 70 & 33.60747 & 117.90060 \\
\hline 198 & 2147 & 37 & 52 & 11 & 75 & 33.60123 & 117.89268 \\
\hline 199 & 2145 & 43 & 47 & 10 & 70 & 33.60382 & 117.88868 \\
\hline 200 & 2146 & 44 & 46 & 10 & 75 & 33.60188 & 117.88743 \\
\hline 201 & 2148 & 53 & 37 & 10 & 75 & 33.59435 & 117.87972 \\
\hline 202 & 2423 & 12 & 65 & 23 & 64 & 32.78068 & 117.24913 \\
\hline 203 & 2424 & 23 & 57 & 20 & 60 & 32.76645 & 117.24747 \\
\hline 204 & 2425 & 21 & 62 & 17 & 62 & 32.76725 & 117.23562 \\
\hline 205 & 2442 & 8 & 62 & 30 & 46 & 32.68920 & 117.23708 \\
\hline 206 & 2441 & 5 & 68 & 27 & 43 & 32.69115 & 117.23802 \\
\hline 207 & 2224 & 16 & 65 & 19 & 50 & 32.71308 & 117.23410 \\
\hline 208 & 2226 & 13 & 70 & 17 & 49 & 32.71112 & 117.23165 \\
\hline 209 & 2435 & 9 & 72 & 19 & 50 & 32.71153 & 117.22292 \\
\hline 210 & 2225 & 11 & 72 & 17 & 51 & 32.71340 & 117.23020 \\
\hline 211 & 2223 & 8 & 75 & 17 & 50 & 32.71542 & 117.23052 \\
\hline 212 & 2222 & 11 & 73 & 16 & 48 & 32.71878 & 117.22585 \\
\hline
\end{tabular}


Table 1 continued

\begin{tabular}{|c|c|c|c|c|c|c|c|}
\hline Map \# & $\begin{array}{l}\text { Sample } \\
\text { Identification }\end{array}$ & $\begin{array}{c}\text { Smectite } \\
(\%)\end{array}$ & $\begin{array}{l}\text { Illite } \\
(\%)\end{array}$ & $\begin{array}{c}\mathrm{K}+\mathrm{C} \\
(\%)\end{array}$ & $\begin{array}{c}\text { Expandable } \\
\text { Layers } \\
(\%)\end{array}$ & $\begin{array}{c}\text { Latitude } \\
\left({ }^{\circ} \mathrm{N}\right)\end{array}$ & $\begin{array}{c}\text { Longitude } \\
\left({ }^{\circ} \mathrm{W}\right)\end{array}$ \\
\hline 213 & 2433 & 13 & 65 & 22 & 55 & 32.72235 & 117.20922 \\
\hline 214 & 2227 & 16 & 66 & 18 & 52 & 32.72373 & 117.20803 \\
\hline 215 & 2221 & 11 & 71 & 18 & 50 & 32.72785 & 117.20512 \\
\hline 216 & 2439 & 11 & 73 & 16 & 52 & 32.72610 & 117.18952 \\
\hline 217 & 2434 & 10 & 69 & 21 & 51 & 32.72490 & 117.18363 \\
\hline 218 & 2228 & 21 & 62 & 17 & 55 & 32.72407 & 117.17817 \\
\hline 219 & 2440 & 12 & 68 & 20 & 56 & 32.71848 & 117.17482 \\
\hline 220 & 2436 & 13 & 68 & 19 & 57 & 32.71503 & 117.18312 \\
\hline 221 & 2263 & 17 & 65 & 18 & 56 & 32.71605 & 117.17598 \\
\hline 222 & 2229 & 17 & 65 & 18 & 50 & 32.70895 & 117.17603 \\
\hline 223 & 2230 & 20 & 64 & 16 & 59 & 32.70253 & 117.17865 \\
\hline 224 & 2251 & 6 & 71 & 23 & 46 & 32.70230 & 117.16207 \\
\hline 225 & 2231 & 20 & 63 & 17 & 59 & 32.69465 & 117.15655 \\
\hline 226 & 2252 & 18 & 63 & 19 & 55 & 32.69187 & 117.15285 \\
\hline 227 & 2233 & 20 & 62 & 18 & 61 & 32.68582 & 117.15183 \\
\hline 228 & 2253 & 21 & 53 & 26 & 65 & 32.68813 & 117.13810 \\
\hline 229 & 2264 & 28 & 56 & 16 & 60 & 32.68538 & 117.13282 \\
\hline 230 & 2239 & 8 & 66 & 26 & 58 & 32.68240 & 117.14010 \\
\hline 231 & 2265 & 25 & 56 & 19 & 58 & 32.68388 & 117.14030 \\
\hline 232 & 2254 & 8 & 65 & 27 & 61 & 32.67725 & 117.16323 \\
\hline 233 & 2255 & 23 & 58 & 19 & 58 & 32.67797 & 117.12940 \\
\hline 234 & 2258 & 18 & 63 & 19 & 70 & 32.67592 & 117.13213 \\
\hline 235 & 2257 & 10 & 68 & 22 & 50 & 32.67683 & 117.13408 \\
\hline 236 & 2256 & 20 & 63 & 17 & 60 & 32.67685 & 117.13587 \\
\hline 237 & 2241 & 17 & 62 & 21 & 61 & 32.67027 & 117.13648 \\
\hline 238 & 2240 & 17 & 64 & 19 & 56 & 32.66753 & 117.15408 \\
\hline 239 & 2259 & 21 & 59 & 20 & 59 & 32.67022 & 117.12473 \\
\hline 240 & 2242 & 16 & 64 & 20 & 60 & 32.66497 & 117.14975 \\
\hline 241 & 2260 & 17 & 62 & 21 & 55 & 32.66718 & 117.12998 \\
\hline 242 & 2243 & 15 & 64 & 21 & 63 & 32.66450 & 117.14265 \\
\hline 243 & 2244 & 8 & 69 & 23 & 51 & 32.65972 & 117.13182 \\
\hline 244 & 2245 & 14 & 64 & 22 & 58 & 32.65083 & 117.14270 \\
\hline 245 & 2262 & 12 & 66 & 22 & 58 & 32.65150 & 117.12293 \\
\hline 246 & 2235 & 9 & 64 & 27 & 55 & 32.64080 & 117.13693 \\
\hline 247 & 2247 & 13 & 63 & 24 & 49 & 32.64233 & 117.12473 \\
\hline 248 & 2238 & 13 & 66 & 21 & 55 & 32.62542 & 117.12865 \\
\hline 249 & 2249 & 14 & 64 & 22 & 53 & 32.62133 & 117.12812 \\
\hline 250 & 2438 & 13 & 68 & 19 & 53 & 32.62230 & 117.10170 \\
\hline
\end{tabular}

${ }^{*}$ weighting factors used are 4 times the illite, 2 times the $\mathrm{k}+\mathrm{c}$, and 1 times the smectite peak areas; $\mathrm{K}+\mathrm{C}$

$=$ kaolinite + chlorite LCMC $=$ low clay mineral content 
Table 2. Un-weighted clay mineral content of the $<2 \mu \mathrm{m}$ size-fraction of Southern California Borderland shelf surface sediments

\begin{tabular}{|c|c|c|c|c|c|}
\hline Map \# & $\begin{array}{l}\text { Sample } \\
\text { Identification }\end{array}$ & $\begin{array}{c}\text { Smectite } \\
(\%)\end{array}$ & $\begin{array}{l}\text { Illite } \\
(\%)\end{array}$ & $\begin{array}{c}\mathrm{K}+\mathrm{C} \\
(\%)\end{array}$ & $\begin{array}{c}\text { Expandable } \\
\text { Layers } \\
(\%)\end{array}$ \\
\hline 1 & 2354 & 56 & 20 & 24 & 50 \\
\hline 2 & 2370 & 66 & 17 & 17 & 56 \\
\hline 3 & 2338 & 50 & 21 & 29 & 50 \\
\hline 4 & 2340 & 48 & 23 & 29 & 49 \\
\hline 5 & 2339 & 44 & 25 & 31 & 55 \\
\hline 6 & 2331 & 66 & 15 & 19 & 60 \\
\hline 7 & 2329 & LCMC & LCMC & LCMC & LCMC \\
\hline 8 & 2330 & 0 & 37 & 63 & 0 \\
\hline 9 & 2328 & 0 & 40 & 60 & 0 \\
\hline 10 & 2375 & 47 & 25 & 28 & 49 \\
\hline 11 & 2376 & 44 & 25 & 31 & 52 \\
\hline 12 & 2129 & 33 & 35 & 32 & 75 \\
\hline 13 & 2130 & 0 & 38 & 62 & 0 \\
\hline 14 & 2131 & 58 & 22 & 20 & 62 \\
\hline 15 & 2132 & 0 & 67 & 33 & 0 \\
\hline 16 & 2273 & 15 & 30 & 55 & 40 \\
\hline 17 & 2275 & 54 & 26 & 20 & 41 \\
\hline 18 & 2274 & 73 & 10 & 17 & 65 \\
\hline 19 & 2276 & 29 & 49 & 22 & 48 \\
\hline 20 & 2341 & 56 & 20 & 24 & 58 \\
\hline 21 & 2342 & 46 & 22 & 32 & 59 \\
\hline 22 & 2310 & 76 & 12 & 12 & 64 \\
\hline 23 & 2308 & 57 & 23 & 20 & 60 \\
\hline 24 & 2377 & LCMC & LCMC & LCMC & LCMC \\
\hline 25 & 2378 & LCMC & LCMC & LCMC & LCMC \\
\hline 26 & 2343 & 46 & 38 & 16 & 47 \\
\hline 27 & 2344 & 76 & 11 & 13 & 70 \\
\hline 28 & 2379 & 65 & 14 & 21 & 55 \\
\hline 29 & S2-97-SC 26B1/0-2 & 71 & 13 & 16 & 70 \\
\hline 30 & 2381 & 68 & 14 & 18 & 67 \\
\hline 31 & 2380 & 77 & 11 & 12 & 62 \\
\hline 32 & S2-97-SC 54B1/0-2 & 75 & 9 & 16 & 56 \\
\hline 33 & S2-97-SC $6 \mathrm{~B} 1 / 0-2$ & 68 & 15 & 17 & 62 \\
\hline 34 & 2382 & 77 & 11 & 12 & 70 \\
\hline 35 & S2-97-SC 4B1/0-2 & 42 & 28 & 30 & 56 \\
\hline 36 & S2-97-SC $24 \mathrm{~B} 1 / 0-2$ & 66 & 16 & 18 & 63 \\
\hline 37 & S2-97-SC 18B1/0-2 & 59 & 18 & 23 & 61 \\
\hline 38 & S2-97-SC $22 B 1 / 0-2$ & 66 & 16 & 18 & 62 \\
\hline 39 & S2-97-SC 20B1/0-2 & 66 & 14 & 20 & 58 \\
\hline 40 & 2305 & 52 & 32 & 16 & 47 \\
\hline 41 & 2307 & LCMC & LCMC & LCMC & LCMC \\
\hline 42 & S2-97-SC 16B1/0-2 & 46 & 25 & 29 & 50 \\
\hline 43 & S2-97-SC 51B1/0-2 & 44 & 24 & 32 & 56 \\
\hline 44 & 2192 & 68 & 12 & 20 & 51 \\
\hline 45 & S2-97-SC 49B1/0-2 & 47 & 32 & 21 & 42 \\
\hline 46 & S2-97-SC 53B3/0-2 & LCMC & LCMC & LCMC & LCMC \\
\hline 47 & S2-97-SC $10 \mathrm{~B} 1 / 0-2$ & 65 & 17 & 18 & 53 \\
\hline 48 & S2-97-SC 52B1/0-2 & 64 & 13 & 23 & 47 \\
\hline 49 & S2-97-SC 50B1/0-2 & 66 & 15 & 19 & 64 \\
\hline 50 & S2-97-SC $40 \mathrm{~B} 1 / 0-2$ & 68 & 15 & 17 & 65 \\
\hline 51 & S2-97-SC $42 B 1 / 0-2$ & 62 & 20 & 18 & 64 \\
\hline 52 & 2306 & 0 & 76 & 24 & 0 \\
\hline 53 & 2384 & 56 & 20 & 24 & 58 \\
\hline
\end{tabular}


Table 2 continued

\begin{tabular}{|c|c|c|c|c|c|}
\hline Map \# & $\begin{array}{l}\text { Sample } \\
\text { Identification }\end{array}$ & $\begin{array}{c}\text { Smectite } \\
(\%)\end{array}$ & $\begin{array}{l}\text { Illite } \\
(\%)\end{array}$ & $\begin{array}{c}\mathrm{K}+\mathrm{C} \\
(\%)\end{array}$ & $\begin{array}{l}\text { Expandable } \\
\text { Layers } \\
(\%)\end{array}$ \\
\hline 54 & S2-97-SC 44B1/0-2 & 48 & 29 & 23 & 50 \\
\hline 55 & 2195 & 76 & 12 & 12 & 65 \\
\hline 56 & $\mathrm{~S} 2-97-\mathrm{SC} \quad 55 \mathrm{~B} 1 / 0-2$ & 59 & 18 & 23 & 43 \\
\hline 57 & 2197 & 70 & 14 & 16 & 61 \\
\hline 58 & $\mathrm{~S} 2-97-\mathrm{SC} \quad 34 \mathrm{~B} 2 / 0-2$ & 64 & 15 & 21 & 65 \\
\hline 59 & 2386 & 12 & 75 & 13 & 41 \\
\hline 60 & S2-97-SC 28B1/0-2 & 67 & 17 & 16 & 52 \\
\hline 61 & S2-97-SC $36 \mathrm{~B} 1 / 0-2$ & 52 & 24 & 24 & 61 \\
\hline 62 & $\mathrm{~S} 2-97-\mathrm{SC} \quad 33 \mathrm{~B} 1 / 0-2$ & 60 & 18 & 22 & 59 \\
\hline 63 & 2387 & 56 & 21 & 23 & 61 \\
\hline 64 & S2-97-SC 48B1/0-2 & 56 & 20 & 24 & 55 \\
\hline 65 & 2200 & 64 & 16 & 20 & 58 \\
\hline 66 & 2201 & 62 & 19 & 19 & 56 \\
\hline 67 & 2202 & 72 & 12 & 16 & 60 \\
\hline 68 & 2389 & LCMC & LCMC & LCMC & LCMC \\
\hline 69 & 2204 & 59 & 20 & 21 & 54 \\
\hline 70 & 2205 & 52 & 24 & 24 & 57 \\
\hline 71 & 2206 & 58 & 20 & 22 & 65 \\
\hline 72 & 2390 & 37 & 26 & 37 & 60 \\
\hline 73 & 2394 & 62 & 21 & 17 & 61 \\
\hline 74 & 2392 & 32 & 44 & 24 & 42 \\
\hline 75 & 2391 & 45 & 29 & 26 & 52 \\
\hline 76 & 2393 & 0 & 64 & 36 & 0 \\
\hline 77 & 2395 & 17 & 51 & 32 & 48 \\
\hline 78 & 2396 & 34 & 30 & 36 & 65 \\
\hline 79 & 2397 & 55 & 21 & 24 & 56 \\
\hline 80 & 2398 & 55 & 15 & 30 & 60 \\
\hline 81 & 2400 & 45 & 27 & 28 & 61 \\
\hline 82 & 2207 & 46 & 25 & 29 & 70 \\
\hline 83 & 2208 & 59 & 18 & 23 & 58 \\
\hline 84 & 2209 & 63 & 19 & 18 & 65 \\
\hline 85 & 2210 & 55 & 20 & 25 & 45 \\
\hline 86 & 2399 & 50 & 24 & 26 & 50 \\
\hline 87 & 2325 & 25 & 45 & 30 & 42 \\
\hline 88 & 2453 & 0 & 42 & 58 & 0 \\
\hline 89 & 2326 & 21 & 55 & 24 & 47 \\
\hline 90 & 2211 & 49 & 25 & 26 & 58 \\
\hline 91 & 2212 & 54 & 23 & 23 & 57 \\
\hline 92 & 2213 & 55 & 20 & 25 & 50 \\
\hline 93 & 2401 & 52 & 25 & 23 & 65 \\
\hline 94 & 2402 & 63 & 24 & 13 & 65 \\
\hline 95 & 2403 & 64 & 21 & 15 & 70 \\
\hline 96 & 2303 & 35 & 31 & 34 & 40 \\
\hline 97 & 2304 & LCMC & LCMC & LCMC & LCMC \\
\hline 98 & 2279 & 70 & 14 & 16 & 70 \\
\hline 99 & 2280 & 58 & 23 & 19 & 70 \\
\hline 100 & 2302 & 62 & 21 & 17 & 70 \\
\hline 101 & 2345 & 62 & 20 & 18 & 73 \\
\hline 102 & 2346 & 65 & 19 & 16 & 75 \\
\hline 103 & 2405 & 58 & 20 & 22 & 55 \\
\hline 104 & 2406 & 51 & 26 & 23 & 53 \\
\hline 105 & 2407 & 41 & 26 & 33 & 53 \\
\hline 106 & 2286 & 52 & 23 & 25 & 65 \\
\hline
\end{tabular}


Table 2 continued

\begin{tabular}{|c|c|c|c|c|c|}
\hline Map \# & $\begin{array}{l}\text { Sample } \\
\text { Identification }\end{array}$ & $\begin{array}{c}\text { Smectite } \\
(\%)\end{array}$ & $\begin{array}{l}\text { Illite } \\
(\%)\end{array}$ & $\begin{array}{c}\mathrm{K}+\mathrm{C} \\
(\%)\end{array}$ & $\begin{array}{c}\text { Expandable } \\
\text { Layers } \\
(\%)\end{array}$ \\
\hline 107 & 2285 & 33 & 30 & 37 & 61 \\
\hline 108 & 2288 & 44 & 27 & 29 & 65 \\
\hline 109 & 2287 & 43 & 30 & 27 & 62 \\
\hline 110 & 2292 & 52 & 26 & 22 & 59 \\
\hline 111 & 2290 & 42 & 30 & 28 & 67 \\
\hline 112 & 2455 & 33 & 45 & 22 & 51 \\
\hline 113 & 2408 & 46 & 32 & 22 & 67 \\
\hline 114 & 2289 & 46 & 27 & 27 & 61 \\
\hline 115 & 2291 & 54 & 25 & 21 & 65 \\
\hline 116 & 2456 & 43 & 29 & 28 & 47 \\
\hline 117 & 2294 & 44 & 29 & 27 & 57 \\
\hline 118 & 2409 & 15 & 65 & 20 & 48 \\
\hline 119 & 2293 & LCMC & LCMC & LCMC & LCMC \\
\hline 120 & 2296 & 41 & 29 & 30 & 53 \\
\hline 121 & 2295 & 40 & 28 & 32 & 60 \\
\hline 122 & 2349 & 56 & 18 & 26 & 65 \\
\hline 123 & 2350 & 38 & 27 & 35 & 70 \\
\hline 124 & 2351 & 38 & 35 & 27 & 56 \\
\hline 125 & 2410 & LCMC & LCMC & LCMC & LCMC \\
\hline 126 & 2411 & 50 & 28 & 22 & 61 \\
\hline 127 & 2412 & 31 & 51 & 18 & 41 \\
\hline 128 & 2215 & 49 & 30 & 21 & 65 \\
\hline 129 & 2216 & 41 & 30 & 29 & 50 \\
\hline 130 & 2219 & 50 & 27 & 23 & 61 \\
\hline 131 & 2220 & 34 & 34 & 32 & 55 \\
\hline 132 & 2414 & 17 & 62 & 21 & 40 \\
\hline 133 & 2415 & 18 & 55 & 27 & 50 \\
\hline 134 & 2417 & 5 & 79 & 16 & 47 \\
\hline 135 & 2418 & 45 & 37 & 18 & 50 \\
\hline 136 & 2419 & 25 & 64 & 11 & 41 \\
\hline 137 & 2353 & 35 & 40 & 25 & 49 \\
\hline 138 & 2352 & 50 & 31 & 19 & 54 \\
\hline 139 & 2335 & 29 & 52 & 19 & 41 \\
\hline 140 & 2169 & 46 & 28 & 26 & 60 \\
\hline 141 & 2174 & 37 & 30 & 33 & 58 \\
\hline 142 & 2178 & 45 & 28 & 27 & 55 \\
\hline 143 & 2184 & 44 & 29 & 27 & 50 \\
\hline 144 & 2170 & 40 & 34 & 26 & 53 \\
\hline 145 & 2176 & 48 & 19 & 33 & 60 \\
\hline 146 & 2182 & 44 & 22 & 34 & 60 \\
\hline 147 & 2428 & 46 & 24 & 30 & 58 \\
\hline 148 & 2168 & 54 & 23 & 23 & 63 \\
\hline 149 & 2421 & 46 & 24 & 30 & 61 \\
\hline 150 & 2172 & 34 & 30 & 36 & 61 \\
\hline 151 & 2177 & 30 & 26 & 44 & 45 \\
\hline 152 & 2162 & 47 & 23 & 30 & 63 \\
\hline 153 & 2173 & 41 & 26 & 33 & 60 \\
\hline 154 & 2300 & 50 & 23 & 27 & 60 \\
\hline 155 & 2299 & 57 & 20 & 23 & 64 \\
\hline 156 & 2297 & 49 & 26 & 25 & 60 \\
\hline 157 & 2298 & 46 & 25 & 29 & 56 \\
\hline 158 & 2427 & 47 & 24 & 29 & 58 \\
\hline 159 & 2426 & 54 & 24 & 22 & 61 \\
\hline
\end{tabular}


Table 2 continued

\begin{tabular}{|c|c|c|c|c|c|}
\hline Map \# & $\begin{array}{l}\text { Sample } \\
\text { Identification }\end{array}$ & $\begin{array}{c}\text { Smectite } \\
(\%)\end{array}$ & $\begin{array}{l}\text { Illite } \\
(\%)\end{array}$ & $\begin{array}{c}\mathrm{K}+\mathrm{C} \\
(\%)\end{array}$ & $\begin{array}{l}\text { Expandable } \\
\text { Layers } \\
(\%)\end{array}$ \\
\hline 160 & 2175 & 47 & 25 & 28 & 61 \\
\hline 161 & 2432 & 40 & 31 & 29 & 56 \\
\hline 162 & 2430 & 51 & 25 & 24 & 57 \\
\hline 163 & 2431 & 47 & 26 & 27 & 57 \\
\hline 164 & 2179 & 33 & 34 & 33 & 55 \\
\hline 165 & 2158 & 48 & 26 & 26 & 59 \\
\hline 166 & 2159 & 51 & 25 & 24 & 63 \\
\hline 167 & 2450 & 69 & 22 & 9 & 53 \\
\hline 168 & 2185 & 35 & 34 & 31 & 58 \\
\hline 169 & 2160 & 43 & 29 & 28 & 54 \\
\hline 170 & 2161 & 53 & 25 & 22 & 65 \\
\hline 171 & 2186 & 44 & 27 & 29 & 59 \\
\hline 172 & 2311 & 70 & 15 & 15 & 62 \\
\hline 173 & 2187 & 55 & 23 & 22 & 60 \\
\hline 174 & 2451 & 67 & 19 & 14 & 57 \\
\hline 175 & 2155 & 43 & 34 & 23 & 55 \\
\hline 176 & 2156 & 64 & 24 & 12 & 63 \\
\hline 177 & 2163 & 44 & 28 & 28 & 58 \\
\hline 178 & 2152 & 41 & 34 & 25 & 50 \\
\hline 179 & 2153 & 50 & 26 & 24 & 61 \\
\hline 180 & 2154 & 53 & 27 & 20 & 60 \\
\hline 181 & 2388 & 58 & 24 & 18 & 63 \\
\hline 182 & 2157 & 38 & 34 & 28 & 50 \\
\hline 183 & 2167 & 57 & 23 & 20 & 63 \\
\hline 184 & 2319 & 58 & 25 & 17 & 62 \\
\hline 185 & 2320 & 34 & 41 & 25 & 46 \\
\hline 186 & 2321 & 17 & 40 & 43 & 51 \\
\hline 187 & 2318 & 61 & 21 & 18 & 65 \\
\hline 188 & 2188 & 53 & 27 & 20 & 61 \\
\hline 189 & 2164 & 50 & 27 & 23 & 62 \\
\hline 190 & 2134 & 52 & 27 & 21 & 50 \\
\hline 191 & 2136 & 67 & 24 & 9 & 64 \\
\hline 192 & 2137 & 70 & 20 & 10 & 65 \\
\hline 193 & 2138 & 66 & 24 & 10 & 67 \\
\hline 194 & 2142 & 76 & 17 & 7 & 70 \\
\hline 195 & 2143 & 79 & 15 & 6 & 75 \\
\hline 196 & 2141 & 72 & 21 & 7 & 75 \\
\hline 197 & 2144 & 72 & 21 & 7 & 70 \\
\hline 198 & 2147 & 67 & 23 & 10 & 75 \\
\hline 199 & 2145 & 71 & 20 & 9 & 70 \\
\hline 200 & 2146 & 72 & 20 & 8 & 75 \\
\hline 201 & 2148 & 79 & 14 & 7 & 75 \\
\hline 202 & 2423 & 29 & 41 & 30 & 64 \\
\hline 203 & 2424 & 48 & 30 & 22 & 60 \\
\hline 204 & 2425 & 46 & 35 & 19 & 62 \\
\hline 205 & 2442 & 20 & 41 & 39 & 46 \\
\hline 206 & 2441 & 12 & 49 & 39 & 43 \\
\hline 207 & 2224 & 39 & 39 & 22 & 50 \\
\hline 208 & 2226 & 33 & 45 & 22 & 49 \\
\hline 209 & 2435 & 25 & 49 & 26 & 50 \\
\hline 210 & 2225 & 30 & 47 & 23 & 51 \\
\hline 211 & 2223 & 24 & 52 & 24 & 50 \\
\hline 212 & 2222 & 29 & 50 & 21 & 48 \\
\hline
\end{tabular}


Table 2 continued

\begin{tabular}{|c|c|c|c|c|c|}
\hline Map \# & $\begin{array}{l}\text { Sample } \\
\text { Identification }\end{array}$ & $\begin{array}{c}\text { Smectite } \\
(\%)\end{array}$ & $\begin{array}{l}\text { Illite } \\
(\%)\end{array}$ & $\begin{array}{l}\mathrm{K}+\mathrm{C} \\
(\%)\end{array}$ & $\begin{array}{c}\text { Expandable } \\
\text { Layers } \\
(\%)\end{array}$ \\
\hline 213 & 2433 & 32 & 41 & 27 & 55 \\
\hline 214 & 2227 & 39 & 39 & 22 & 52 \\
\hline 215 & 2221 & 30 & 46 & 24 & 50 \\
\hline 216 & 2439 & 29 & 50 & 21 & 52 \\
\hline 217 & 2434 & 28 & 45 & 27 & 51 \\
\hline 218 & 2228 & 46 & 35 & 19 & 55 \\
\hline 219 & 2440 & 30 & 44 & 26 & 56 \\
\hline 220 & 2436 & 32 & 44 & 24 & 57 \\
\hline 221 & 2263 & 40 & 39 & 21 & 56 \\
\hline 222 & 2229 & 40 & 39 & 21 & 50 \\
\hline 223 & 2230 & 45 & 36 & 19 & 59 \\
\hline 224 & 2251 & 15 & 51 & 34 & 46 \\
\hline 225 & 2231 & 45 & 35 & 20 & 59 \\
\hline 226 & 2252 & 42 & 37 & 21 & 55 \\
\hline 227 & 2233 & 45 & 35 & 20 & 61 \\
\hline 228 & 2253 & 44 & 28 & 28 & 65 \\
\hline 229 & 2264 & 56 & 28 & 16 & 60 \\
\hline 230 & 2239 & 21 & 44 & 35 & 58 \\
\hline 231 & 2265 & 52 & 29 & 19 & 58 \\
\hline 232 & 2254 & 21 & 43 & 36 & 61 \\
\hline 233 & 2255 & 48 & 31 & 21 & 58 \\
\hline 234 & 2258 & 42 & 36 & 22 & 70 \\
\hline 235 & 2257 & 26 & 45 & 29 & 50 \\
\hline 236 & 2256 & 44 & 36 & 20 & 60 \\
\hline 237 & 2241 & 39 & 36 & 25 & 61 \\
\hline 238 & 2240 & 40 & 37 & 23 & 56 \\
\hline 239 & 2259 & 47 & 32 & 21 & 59 \\
\hline 240 & 2242 & 39 & 38 & 23 & 60 \\
\hline 241 & 2260 & 39 & 36 & 25 & 55 \\
\hline 242 & 2243 & 36 & 38 & 26 & 63 \\
\hline 243 & 2244 & 22 & 46 & 32 & 51 \\
\hline 244 & 2245 & 35 & 38 & 27 & 58 \\
\hline 245 & 2262 & 30 & 42 & 28 & 58 \\
\hline 246 & 2235 & 23 & 42 & 35 & 55 \\
\hline 247 & 2247 & 32 & 39 & 29 & 49 \\
\hline 248 & 2238 & 32 & 42 & 26 & 55 \\
\hline 249 & 2249 & 34 & 39 & 27 & 53 \\
\hline 250 & 2438 & 33 & 43 & 24 & 53 \\
\hline
\end{tabular}

$\mathrm{K}+\mathrm{C}=$ kaolinite + chlorite LCMC $=$ low clay mineral content 
Table 3. Clay mineral content of samples with depth in cores from Santa Monica Bay; core locations and surface sediment data for the same cores are listed in table 1

\begin{tabular}{|c|c|c|c|c|c|}
\hline Map \# & $\begin{array}{l}\text { Sample Identification/ } \\
\text { sample interval in } \mathrm{cm}\end{array}$ & $\begin{array}{l}\text { Smectite } \\
(\%)\end{array}$ & $\begin{array}{l}\text { Illite } \\
(\%)\end{array}$ & $\begin{array}{l}\mathrm{K}+\mathrm{C} \\
(\%)\end{array}$ & $\begin{array}{c}\text { Ex Layers } \\
(\%)\end{array}$ \\
\hline 29 & S2-97-SC 26B1/4-6 & 58 & 28 & 14 & 63 \\
\hline 29 & S2-97-SC 26B1/10-12 & 44 & 38 & 18 & 69 \\
\hline 29 & S2-97-SC 26B1/18-20 & 40 & 40 & 20 & 69 \\
\hline 29 & S2-97-SC 26B1/24-26 & 38 & 50 & 12 & 49 \\
\hline 32 & $\mathrm{~S} 2-97-\mathrm{SC} \quad 54 \mathrm{~B} 1 / 18-20$ & LCMC & LCMC & LCMC & LCMC \\
\hline 32 & $\mathrm{~S} 2-97-\mathrm{SC} \quad 54 \mathrm{~B} 1 / 32-34$ & 46 & 34 & 20 & 55 \\
\hline 33 & S2-97-SC 6B1/10-12 & 45 & 30 & 25 & 65 \\
\hline 33 & S2-97-SC 6B1/22-24 & 56 & 26 & 18 & 75 \\
\hline 35 & S2-97-SC 4B1/4-6 & 31 & 43 & 26 & 41 \\
\hline 35 & S2-97-SC 4B1/10-12 & 26 & 49 & 25 & 55 \\
\hline 35 & S2-97-SC 4B1/14-16 & (31) & (49) & (20) & (41) \\
\hline 36 & S2-97-SC 24B1/4-6 & (40) & (41) & (19) & (48) \\
\hline 36 & S2-97-SC 24B1/10-12 & 53 & 31 & 16 & 70 \\
\hline 36 & S2-97-SC 24B1/20-22 & 46 & 33 & 21 & 70 \\
\hline 36 & S2-97-SC 24B1/30-32 & 52 & 31 & 17 & 69 \\
\hline 37 & S2-97-SC 18B1/6-8 & 32 & 47 & 21 & 50 \\
\hline 37 & S2-97-SC $18 B 1 / 12-14$ & LCMC & LCMC & LCMC & LCMC \\
\hline 37 & S2-97-SC $18 \mathrm{~B} 1 / 18-20$ & LCMC & LCMC & LCMC & LCMC \\
\hline 37 & S2-97-SC $18 \mathrm{~B} 1 / 24-26$ & 19 & 53 & 28 & 41 \\
\hline 37 & S2-97-SC $18 \mathrm{~B} 1 / 26-28$ & 49 & 36 & 15 & 50 \\
\hline 38 & S2-97-SC 22B1/10-12 & (29) & (57) & (14) & (44) \\
\hline 38 & S2-97-SC 22B1/18-20 & 29 & 51 & 20 & 49 \\
\hline 39 & S2-97-SC 20B1/4-6 & 31 & 43 & 26 & 50 \\
\hline 39 & S2-97-SC 20B1/10-12 & (28) & (47) & (25) & (44) \\
\hline 39 & S2-97-SC 20B1/18-20 & 31 & 47 & 22 & 59 \\
\hline 39 & S2-97-SC 20B1/20-22 & LCMC & LCMC & LCMC & LCMC \\
\hline 39 & S2-97-SC 20B1/22-24 & 21 & 58 & 21 & 48 \\
\hline 42 & S2-97-SC 16B1/4-6 & 23 & 51 & 26 & 50 \\
\hline 42 & S2-97-SC 16B1/8-10 & 40 & 37 & 23 & 62 \\
\hline 42 & S2-97-SC 16B1/16-18 & 30 & 48 & 22 & 60 \\
\hline 42 & S2-97-SC $16 \mathrm{~B} 1 / 26-28$ & 33 & 45 & 22 & 65 \\
\hline 43 & S2-97-SC 51B1/2-4 & (31) & (45) & (24) & (44) \\
\hline 43 & S2-97-SC 51B1/4-6 & 28 & 40 & 32 & 50 \\
\hline 43 & S2-97-SC 51B1/10-12 & 25 & 50 & 25 & 50 \\
\hline 43 & S2-97-SC 51B1/16-18 & 29 & 50 & 21 & 50 \\
\hline 43 & S2-97-SC 51B1/20-22 & 32 & 45 & 23 & 62 \\
\hline 45 & S2-97-SC 49B1/14-16 & 40 & 34 & 26 & 53 \\
\hline 45 & S2-97-SC 49B1/22-24 & 37 & 44 & 19 & 59 \\
\hline 46 & S2-97-SC 53B3/24-26 & LCMC & LCMC & LCMC & LCMC \\
\hline 46 & S2-97-SC 53B3/42-44 & $(27)$ & (47) & (26) & (41) \\
\hline 47 & S2-97-SC 10B1/10-12 & LCMC & LCMC & LCMC & LCMC \\
\hline 47 & S2-97-SC $10 \mathrm{~B} 1 / 22-24$ & LCMC & LCMC & LCMC & LCMC \\
\hline 47 & S2-97-SC 10B1/36-38 & 36 & 42 & 22 & 55 \\
\hline 47 & S2-97-SC 10B1/40-42 & 41 & 37 & 22 & 55 \\
\hline 47 & S2-97-SC 10B1/42-44 & 33 & 45 & 22 & 50 \\
\hline 48 & S2-97-SC 52B1/2-4 & 26 & 53 & 21 & 40 \\
\hline 48 & S2-97-SC 52B1/4-6 & 15 & 59 & 26 & 41 \\
\hline 48 & S2-97-SC 52B1/8-10 & (39) & (42) & (19) & (45) \\
\hline 48 & S2-97-SC 52B1/22-24 & 46 & 33 & 21 & 50 \\
\hline 49 & S2-97-SC 50B1/6-8 & (33) & (46) & (21) & (40) \\
\hline 49 & S2-97-SC 50B1/16-18 & (27) & (51) & (22) & (44) \\
\hline 49 & S2-97-SC 50B1/22-24 & 29 & 42 & 29 & 50 \\
\hline 50 & S2-97-SC 40B1/8-10 & 34 & 41 & 25 & 56 \\
\hline
\end{tabular}


Table 3 continued

\begin{tabular}{llcccc}
\hline Map \# & $\begin{array}{l}\text { Sample Identification/ } \\
\text { sample interval in cm }\end{array}$ & $\begin{array}{c}\text { Smectite } \\
(\%)\end{array}$ & $\begin{array}{c}\text { Illite } \\
(\%)\end{array}$ & $\begin{array}{c}\text { K+C } \\
(\%)\end{array}$ & $\begin{array}{c}\text { Ex Layers } \\
(\%)\end{array}$ \\
\hline 50 & S2-97-SC 40B1/18-20 & $(34)$ & $(44)$ & $(22)$ & $(45)$ \\
51 & S2-97-SC 42B1/4-6 & $(31)$ & $(53)$ & $(16)$ & $(44)$ \\
51 & S2-97-SC 42B1/10-12 & 27 & 47 & 26 & 53 \\
51 & S2-97-SC 42B1/20-22 & 23 & 53 & 24 & 50 \\
54 & S2-97-SC 44B1/8-10 & 26 & 52 & 22 & - \\
54 & S2-97-SC 44B1/18-20 & 31 & 49 & 20 & 44 \\
56 & S2-97-SC 55B1/10-12 & 34 & 41 & 25 & 59 \\
56 & S2-97-SC 55B1/24-26 & $(32)$ & $(47)$ & $(21)$ & $(42)$ \\
58 & S2-97-SC 34B2/2-4 & $(29)$ & $(41)$ & $(30)$ & $(41)$ \\
58 & S2-97-SC 34B2/6-8 & 30 & 45 & 25 & 50 \\
58 & S2-97-SC 34B2/14-16 & 11 & 56 & 33 & 50 \\
58 & S2-97-SC 34B2/26-28 & 28 & 46 & 26 & 55 \\
60 & S2-97-SC 28B1/2-4 & 37 & 40 & 23 & 48 \\
60 & S2-97-SC 28B1/8-10 & 39 & 38 & 23 & 50 \\
60 & S2-97-SC 28B1/14-16 & 37 & 42 & 21 & 50 \\
60 & S2-97-SC 28B1/24-26 & 24 & 49 & 27 & 50 \\
61 & S2-97-SC 36B1/2-4 & 38 & 36 & 26 & 46 \\
61 & S2-97-SC 36B1/6-8 & 30 & 45 & 25 & 65 \\
61 & S2-97-SC 36B1/10-12 & 38 & 39 & 23 & 60 \\
61 & S2-97-SC 36B1/20-22 & $(42)$ & $(37)$ & $(21)$ & $(44)$ \\
62 & S2-97-SC 33B1/4-6 & 45 & 40 & 15 & 46 \\
62 & S2-97-SC 33B1/8-10 & $(50)$ & $(33)$ & $(17)$ & $(42)$ \\
64 & S2-97-SC 48B1/4-6 & 37 & 38 & 25 & 49 \\
64 & S2-97-SC 48B1/8-10 & 29 & 42 & 29 & 51 \\
64 & S2-97-SC 48B1/16-18 & 33 & 43 & 24 & 58 \\
64 & S2-97-SC 48B1/28-30 & 27 & 49 & 24 & 55 \\
\hline
\end{tabular}

$\mathrm{k}+\mathrm{c}$ is kaolinite plus chlorite; Ex is expandable, which represents the percent smectite layers in the smectite-illite mixed layer phase, listed simply as smectite in column 3; LCMC is low clay mineral content, below the amount needed for quantification; numbers in parenthesis are samples with LCMC, but with mineral contents that are comparable with those of adjacent sample(s) 
Table 4. Clay mineral content of Southern California river samples and Palos Verdes Headland (first 6) samples. Rivers listed from north to south.

\begin{tabular}{|c|c|c|c|c|}
\hline $\begin{array}{l}\text { Sample } \\
\text { Identification }\end{array}$ & $\begin{array}{c}\text { Smectite } \\
(\%)\end{array}$ & $\begin{array}{l}\text { Illite } \\
(\%)\end{array}$ & $\begin{array}{c}\mathrm{K}+\mathrm{C} \\
(\%)\end{array}$ & $\begin{array}{c}\text { Expandable } \\
\text { layers }(\%)\end{array}$ \\
\hline $05-1 C-10-99$ & 44 & 21 & 35 & 70 \\
\hline 06-1E-10-99 & 100 & 0 & 0 & 50 \\
\hline $06-3 B-10-99$ & 43 & 35 & 22 & 58 \\
\hline $06-3 C-10-99$ & 87 & 13 & 0 & 49 \\
\hline 06-3D-10-99 & 100 & 0 & 0 & 52 \\
\hline 06-3E-10-99 & 86 & 7 & 7 & 65 \\
\hline Santa Ynez R. 1975B & 28 & 38 & 34 & -- \\
\hline Santa Ynez R. 061300 B & 20 & 41 & 39 & 50 \\
\hline Ventura R. $061300 \mathrm{~W}$ & $\mathrm{LCMC}$ & $\mathrm{LCMC}$ & $\mathrm{LCMC}$ & LCMC \\
\hline Ventura R. 061300 B & 22 & 50 & 28 & 56 \\
\hline Santa Clara R. 061400 B & 42 & 41 & 17 & 55.5 \\
\hline Calleguas R. $061300 \mathrm{~W}$ & LCMC & $\mathrm{LCMC}$ & $\mathrm{LCMC}$ & LCMC \\
\hline Calleguas R. 061300 B & 50 & 38 & 12 & 62.5 \\
\hline Malibu Cr $022400 \mathrm{~W}$ & 73 & 19 & 8 & 75 \\
\hline Malibu Cr $041800 \mathrm{~W}$ & 64 & 29 & 7 & 70 \\
\hline Malibu R. 061300 W & LCMC & $\mathrm{LCMC}$ & $\mathrm{LCMC}$ & LCMC \\
\hline Malibu R. 061300 B & $\mathrm{LCMC}$ & $\mathrm{LCMC}$ & $\mathrm{LCMC}$ & LCMC \\
\hline Ballona $\mathrm{Cr} 022400 \mathrm{~W}$ & 41 & 43 & 16 & 62 \\
\hline Ballona $\mathrm{Cr} 022800 \mathrm{~W}$ & 43 & 38 & 19 & 59 \\
\hline Ballona $\mathrm{Cr} 041800 \mathrm{~W}$ & 40 & 43 & 17 & 59.5 \\
\hline LA River $022400 \mathrm{~W}$ & 22 & 55 & 23 & 55 \\
\hline LA River $022800 \mathrm{~W}$ & 0 & 62 & 38 & 0 \\
\hline LA River $041900 \mathrm{~W}$ & 20 & 57 & 23 & 46 \\
\hline LA River $061300 \mathrm{~W}$ & 47 & 40 & 13 & 64.5 \\
\hline LA River 061300 B & 42 & 44 & 14 & 55 \\
\hline San Gabriel R $022400 \mathrm{~W}$ & 30 & 52 & 18 & 65 \\
\hline San Gabriel R $022800 \mathrm{~W}$ & 49 & 36 & 15 & 78 \\
\hline San Gabriel R 041900 W & 33 & 53 & 14 & 65 \\
\hline San Gabriel R. $061400 \mathrm{~W}$ & LCMC & LCMC & LCMC & LCMC \\
\hline San Gabriel R. 061400 B & 22 & 57 & 21 & 51 \\
\hline Coyote $\mathrm{Cr} 022400 \mathrm{~W}$ & 10 & 64 & 26 & 50 \\
\hline Coyote $\mathrm{Cr} 022800 \mathrm{~W}$ & 28 & 54 & 18 & 52 \\
\hline Coyote Cr $041900 \mathrm{~W}$ & LCMC & LCMC & $\mathrm{LCMC}$ & LCMC \\
\hline Santa Ana R 020998 W & 22 & 52 & 26 & 70 \\
\hline Santa Ana R. 061400 B & 37 & 41 & 22 & 59 \\
\hline San Juan Cr. 061400 B & 28 & 69 & 3 & 65 \\
\hline S. Margarita R. $061400 \mathrm{~W}$ & 6 & 74 & 20 & 43 \\
\hline S. Margarita R. 061400 B & 16 & 61 & 23 & 55 \\
\hline San Luis Rey R. 061400 W & LCMC & LCMC & $\mathrm{LCMC}$ & LCMC \\
\hline San Luis Rey R. 061400 B & 5 & 51 & 44 & 41 \\
\hline San Diego R. $061400 \mathrm{~W}$ & 4 & 75 & 21 & 50 \\
\hline San Diego R. 061400 B & 63 & 29 & 8 & 64.5 \\
\hline Chollas Cr 021200 W & 32 & 48 & 20 & 65 \\
\hline
\end{tabular}

05-1C is composite of Monterey Fm; 06-1E is sediment produced from weathering of basalt; 06-3B-3E are from toe of Portuguese Bend Landslide; numbers following river names are dates of collection and $\mathrm{W}$ is suspended sediment and B is bottom sediment; the first sample from Santa Ynez R. is from Griggs and Hein (1980); LCMC means low clay mineral content, below semi-quantifiable limits 
Table 5. Duplicate sample (with the asterix) analyses used to calculate precision

\begin{tabular}{|c|c|c|c|c|c|}
\hline $\begin{array}{l}\text { Map } \\
\#\end{array}$ & Sample Identification & $\begin{array}{c}\text { Smectite } \\
(\%)\end{array}$ & $\begin{array}{c}\text { Illite } \\
(\%)\end{array}$ & $\begin{array}{c}\mathrm{K}+\mathrm{C} \\
(\%)\end{array}$ & $\begin{array}{c}\text { Expandable } \\
\text { Layers } \\
(\%)\end{array}$ \\
\hline 14 & 2131 & 32 & 47 & 21 & 62 \\
\hline 14 & $2131 *$ & 29 & 48 & 23 & 55 \\
\hline 24 & 2377 & (46) & (22) & (32) & (44) \\
\hline 24 & $2377 *$ & 42 & 32 & 26 & 50 \\
\hline 25 & 2378 & (60) & (21) & (19) & $(50)$ \\
\hline 25 & $2378 *$ & 58 & 21 & 21 & 44 \\
\hline 32 & S2-97-SC 54B1/0-2 & 53 & 25 & 22 & 56 \\
\hline 32 & S2-97-SC 54B1/0-2* & 51 & 26 & 23 & 56 \\
\hline 72 & 2390 & 17 & 48 & 35 & 60 \\
\hline 72 & $2390 *$ & 22 & 46 & 32 & 63 \\
\hline 73 & 2394 & 34 & 47 & 19 & 61 \\
\hline 73 & $2394 *$ & 31 & 48 & 21 & 60 \\
\hline 79 & 2397 & 29 & 45 & 26 & 56 \\
\hline 79 & $2397 *$ & 26 & 48 & 26 & 69 \\
\hline 81 & 2400 & 22 & 52 & 26 & 61 \\
\hline 81 & $2400 *$ & 29 & 48 & 23 & 65 \\
\hline 98 & 2279 & 45 & 35 & 20 & 70 \\
\hline 98 & $2279 *$ & 51 & 31 & 18 & 75 \\
\hline 108 & 2288 & 22 & 51 & 27 & 65 \\
\hline 108 & $2288 *$ & 17 & 55 & 28 & 67 \\
\hline 124 & 2351 & 16 & 61 & 23 & 56 \\
\hline 124 & $2351 *$ & 17 & 58 & 25 & 50 \\
\hline 131 & 2220 & 15 & 58 & 27 & 55 \\
\hline 131 & $2220 *$ & 22 & 57 & 21 & 63 \\
\hline 143 & 2184 & 21 & 54 & 25 & 50 \\
\hline 143 & $2184 *$ & 22 & 56 & 22 & 59 \\
\hline 144 & 2170 & 18 & 59 & 23 & 53 \\
\hline 144 & $2170 *$ & 15 & 62 & 23 & 55 \\
\hline 146 & 2182 & 22 & 45 & 33 & 60 \\
\hline 146 & $2182^{*}$ & 22 & 45 & 33 & 61 \\
\hline 180 & 2154 & 26 & 54 & 20 & 60 \\
\hline 180 & $2154 *$ & 27 & 53 & 20 & 58 \\
\hline 192 & 2137 & 41 & 48 & 11 & 65 \\
\hline 192 & $2137 *$ & 34 & 52 & 14 & 58 \\
\hline 198 & 2147 & 37 & 52 & 11 & 75 \\
\hline 198 & $2147 *$ & 36 & 51 & 13 & 75 \\
\hline 202 & 2423 & 12 & 65 & 23 & 64 \\
\hline 202 & $2423 *$ & 14 & 66 & 20 & 69 \\
\hline 208 & 2226 & 13 & 70 & 17 & 49 \\
\hline 208 & $2226 *$ & 13 & 68 & 19 & 56 \\
\hline 224 & 2251 & 6 & 71 & 23 & 46 \\
\hline 224 & $2251 *$ & 10 & 66 & 24 & 57 \\
\hline 241 & 2260 & 17 & 62 & 21 & 55 \\
\hline 241 & $2260 *$ & 9 & 64 & 27 & 54 \\
\hline
\end{tabular}

$\mathrm{K}+\mathrm{C}=$ kaolinite + chlorite 


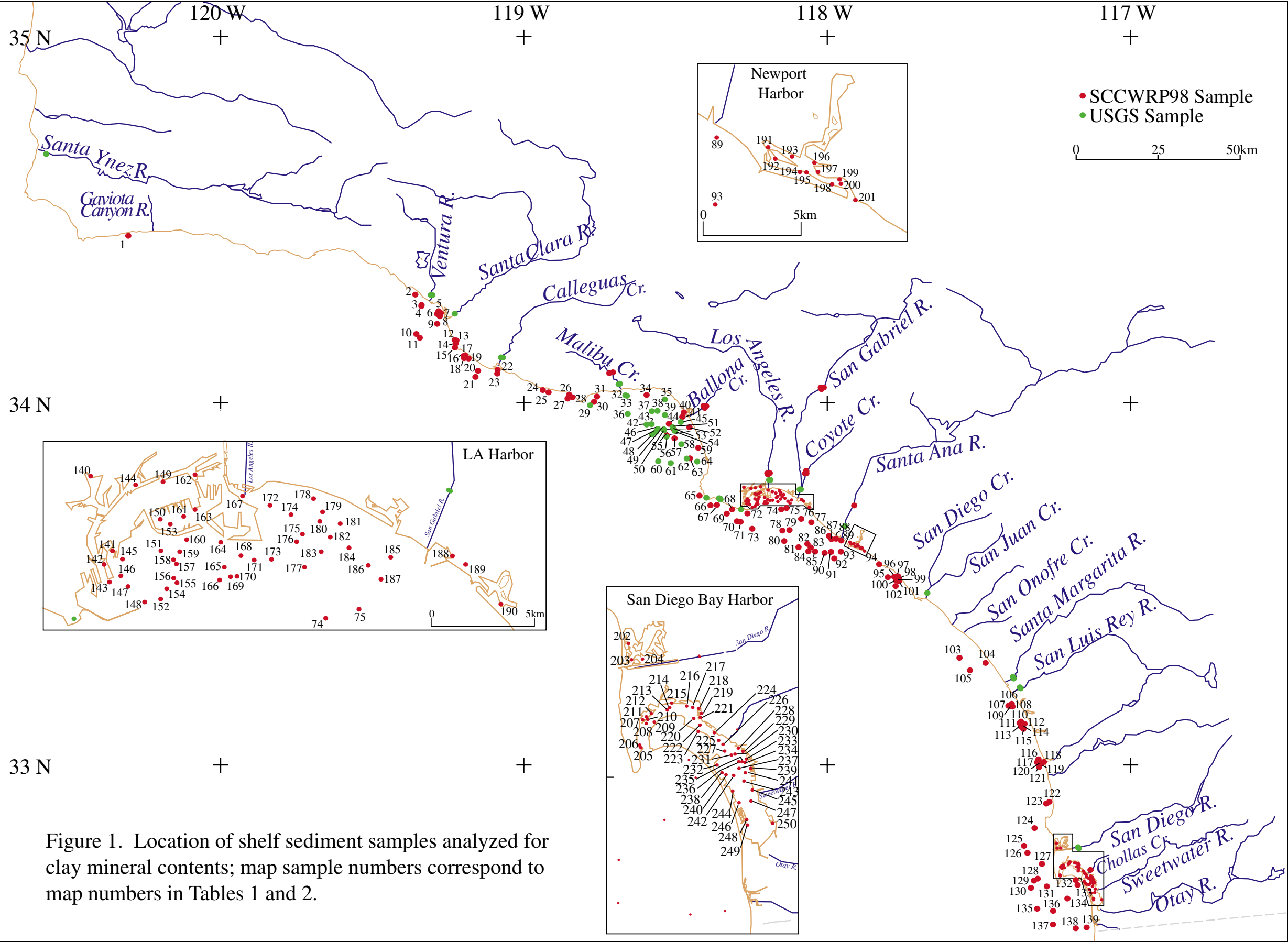




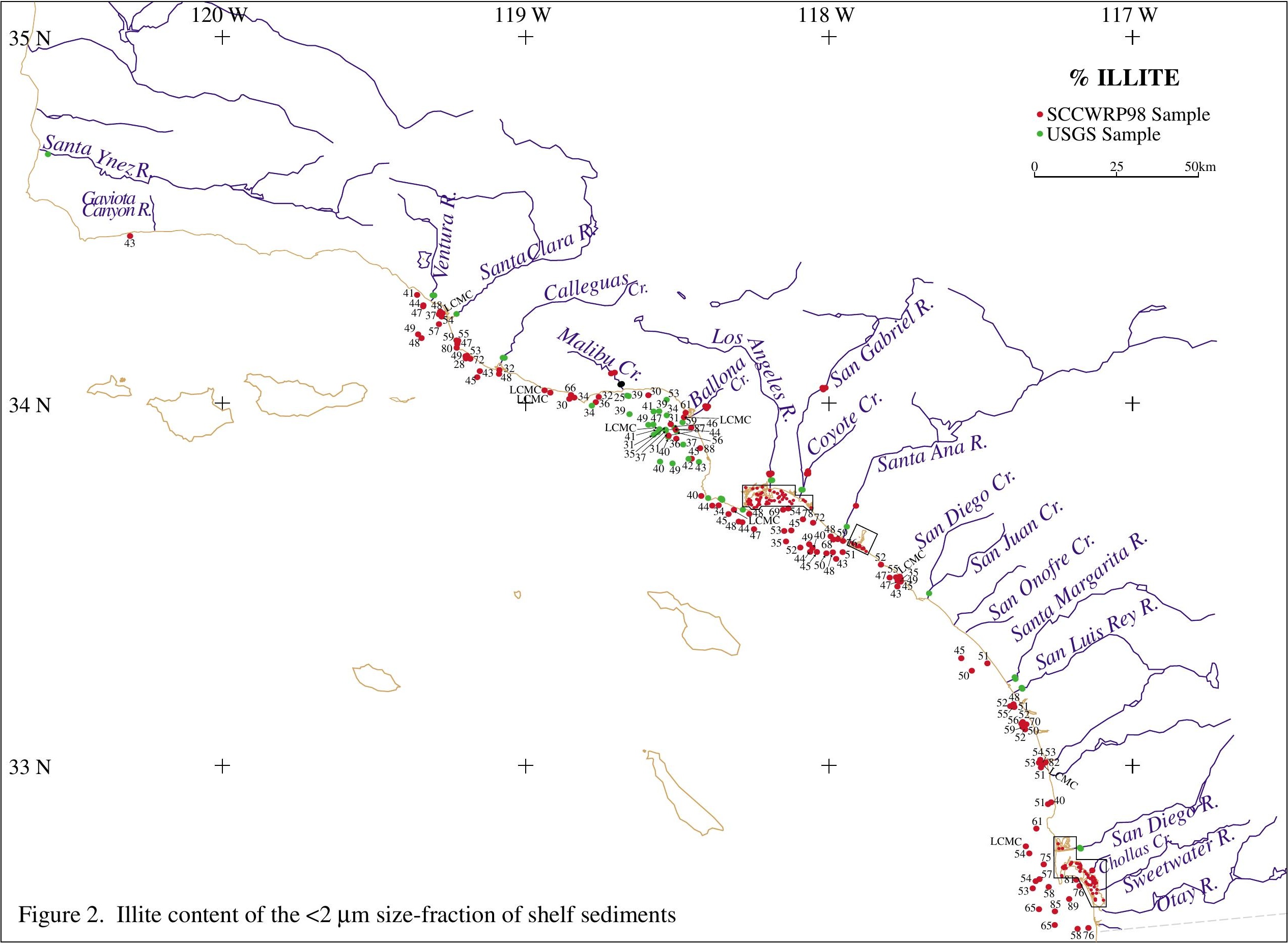




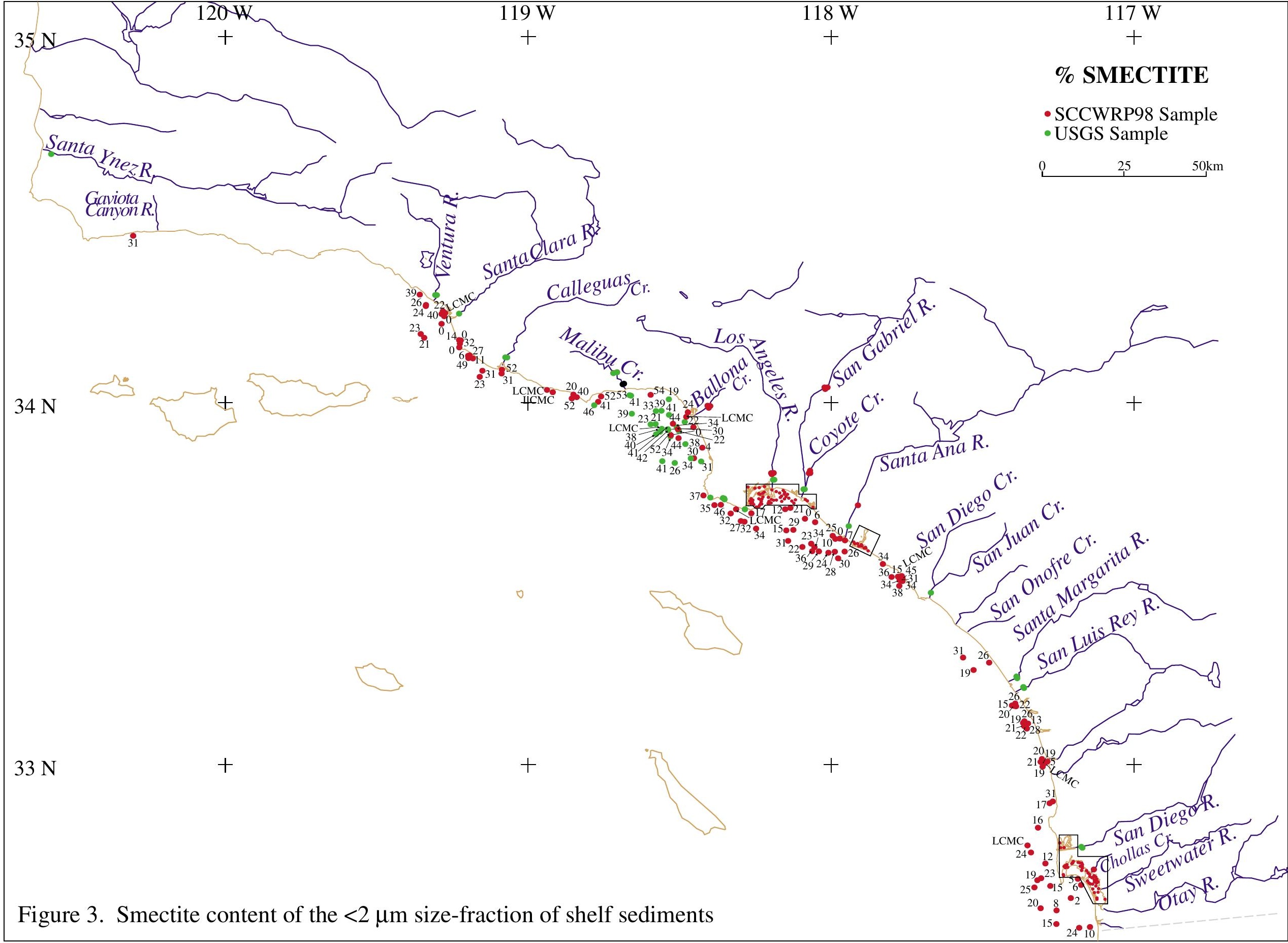




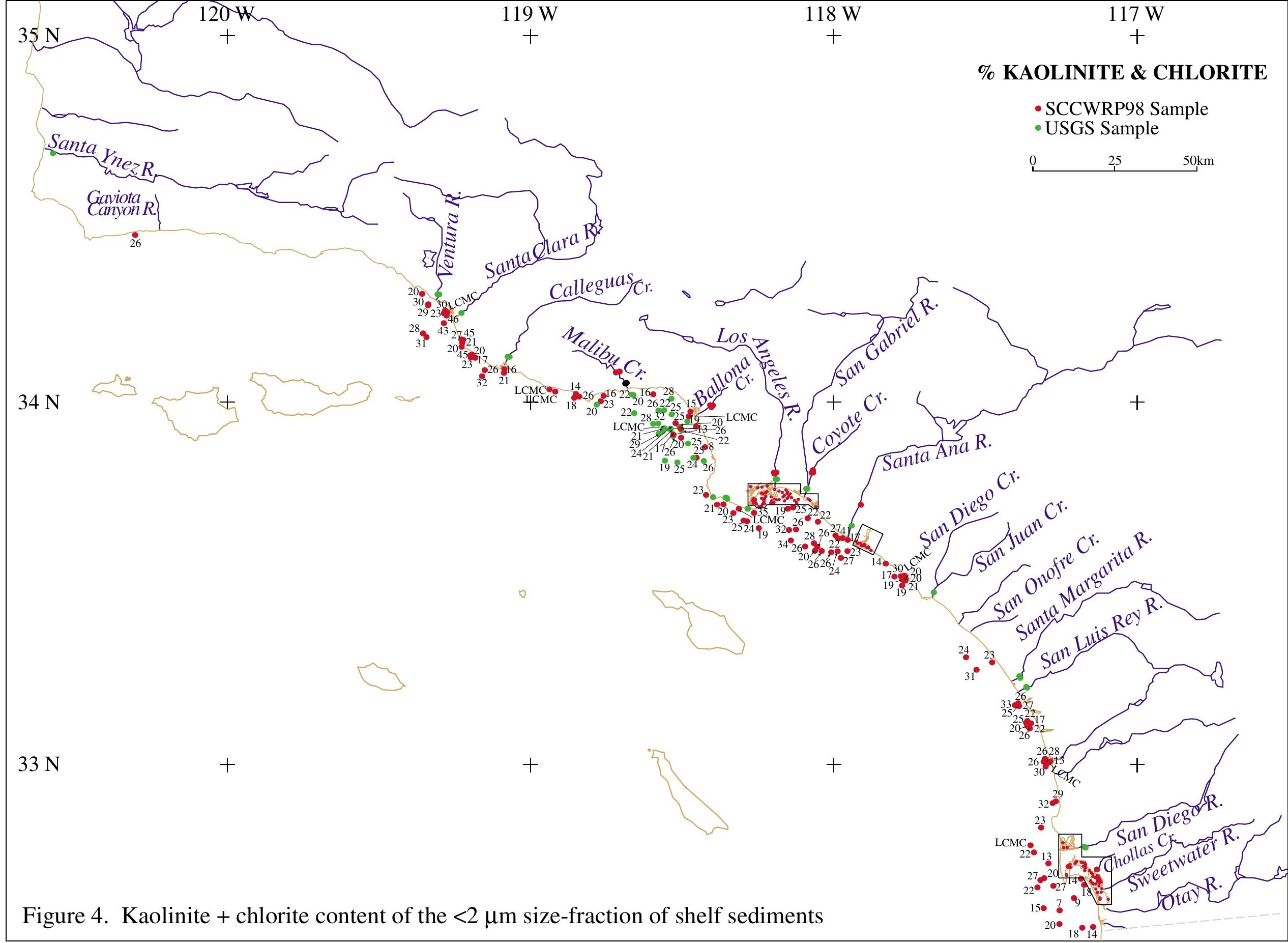




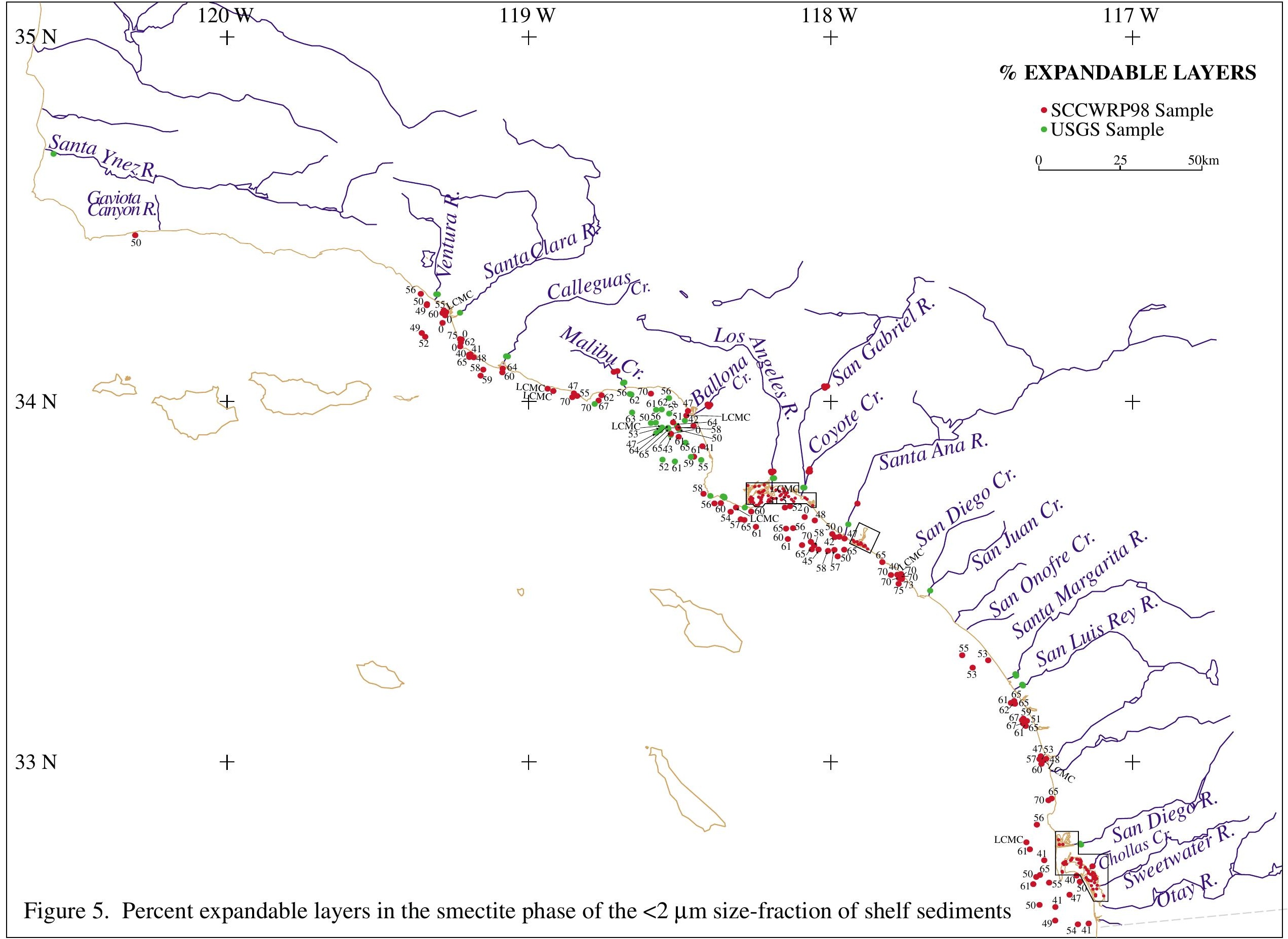




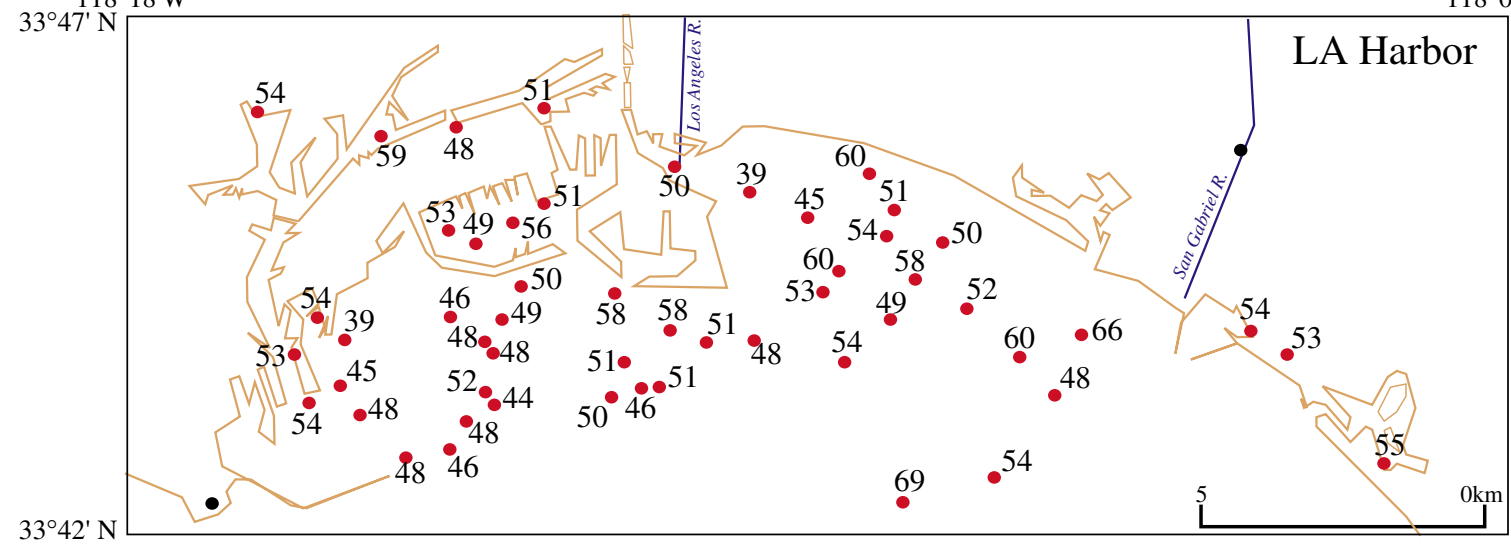

$117^{\circ} 58^{\prime} \mathrm{W}$

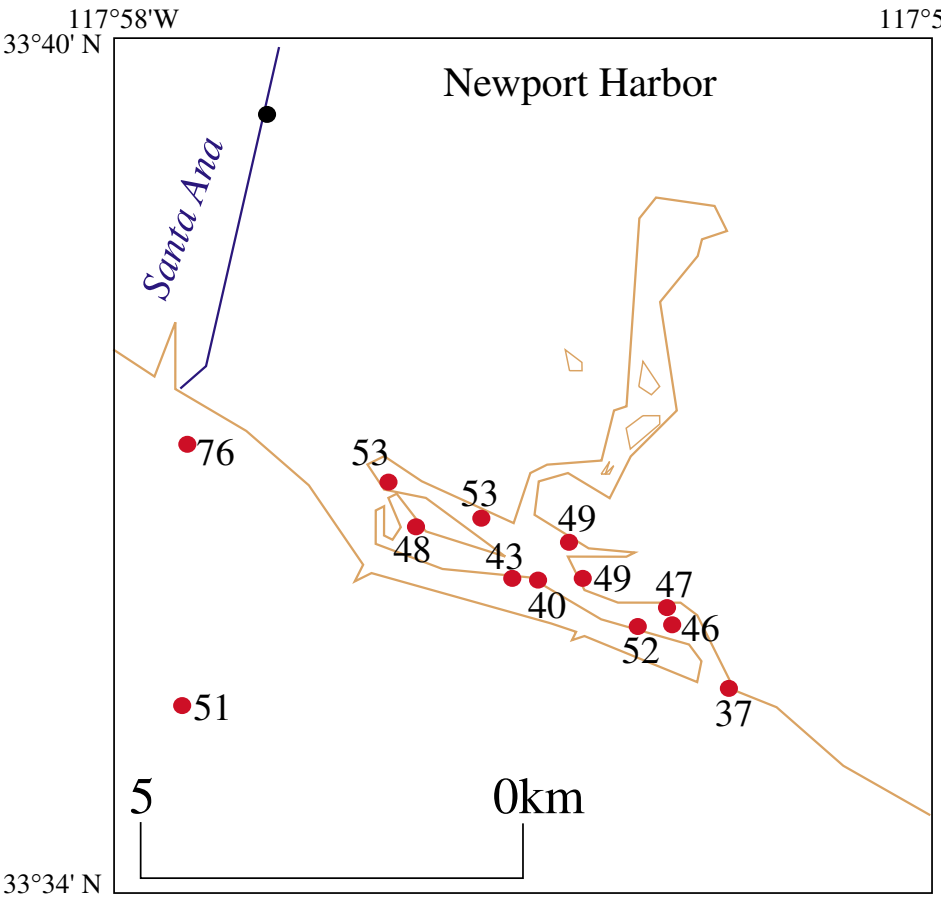

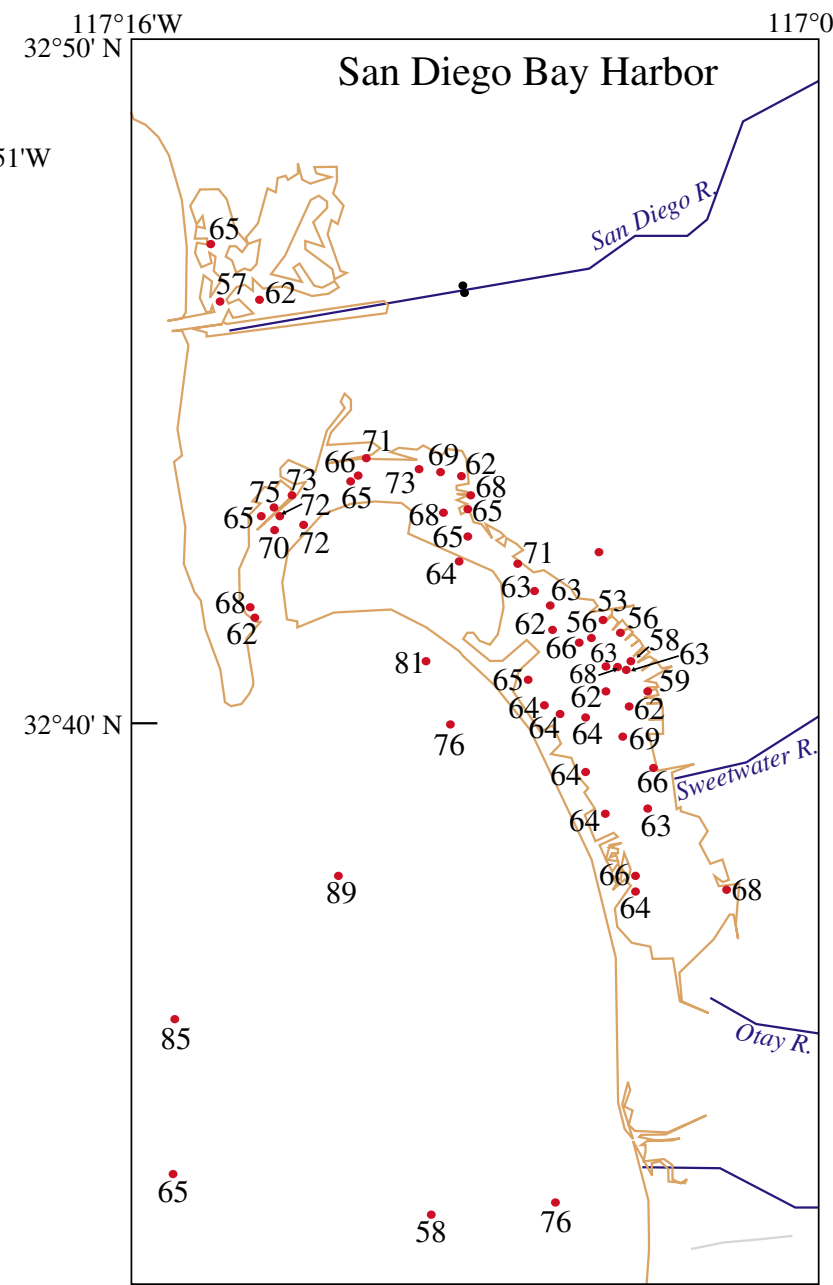

Figure 6. Illite content of the $<2 \mu \mathrm{m}$ size-fraction of harbor sediments 


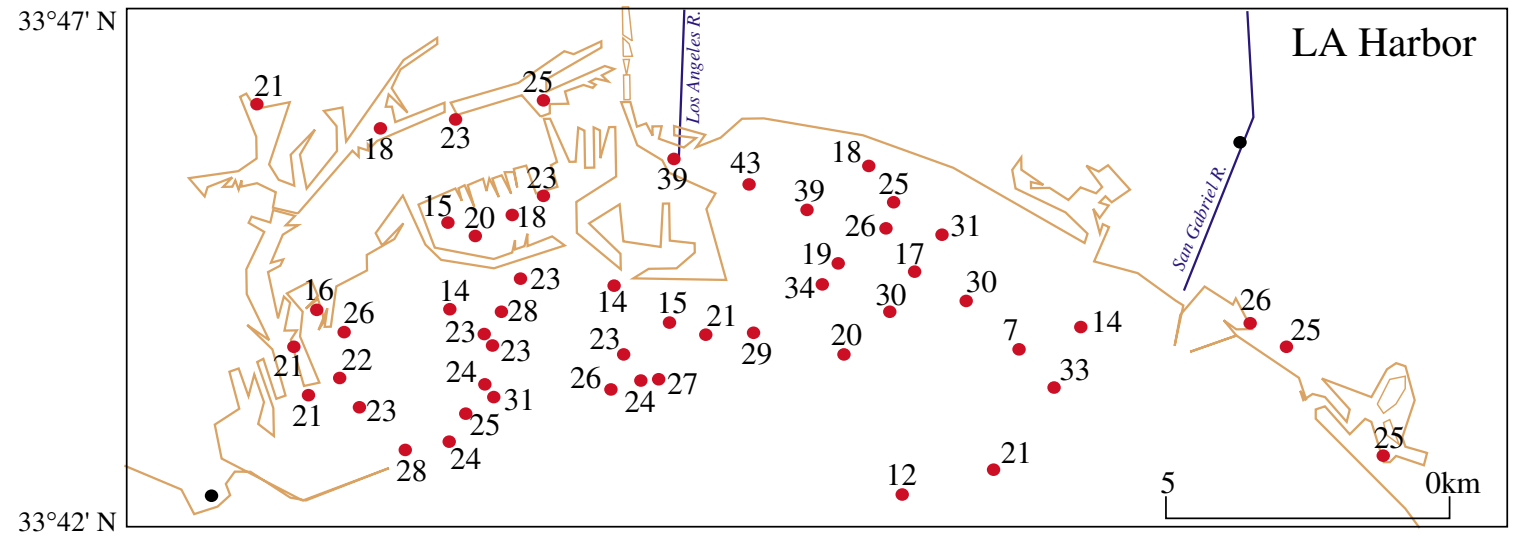

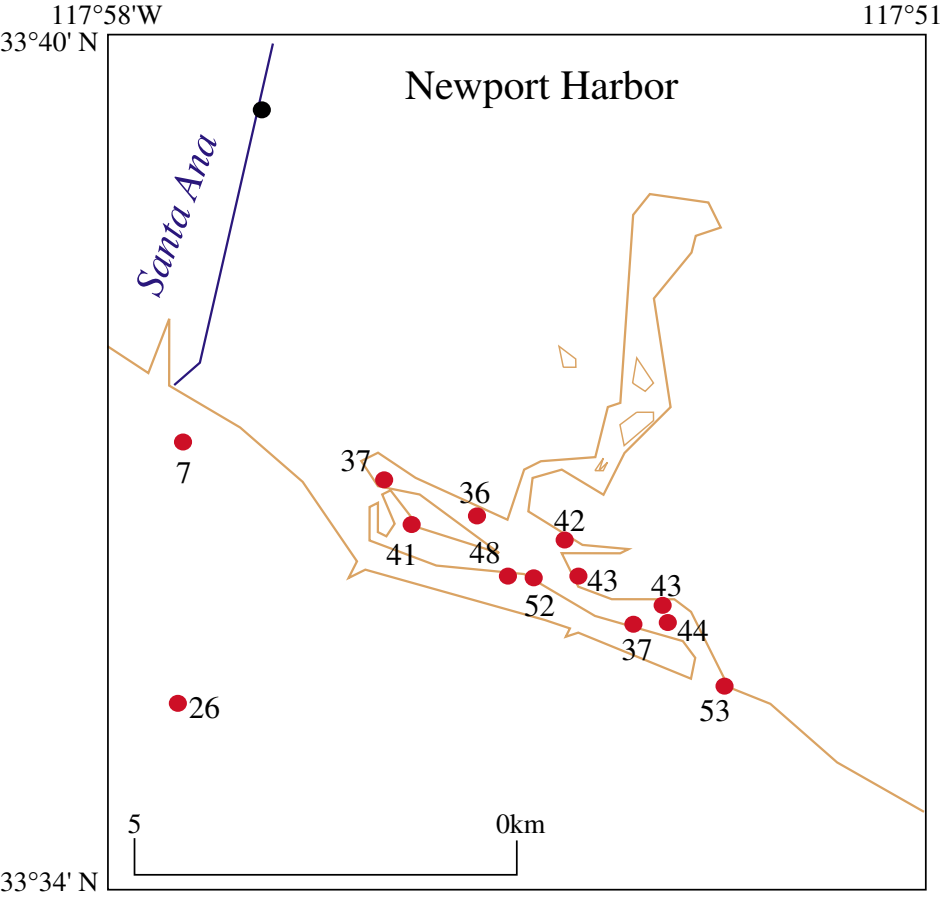

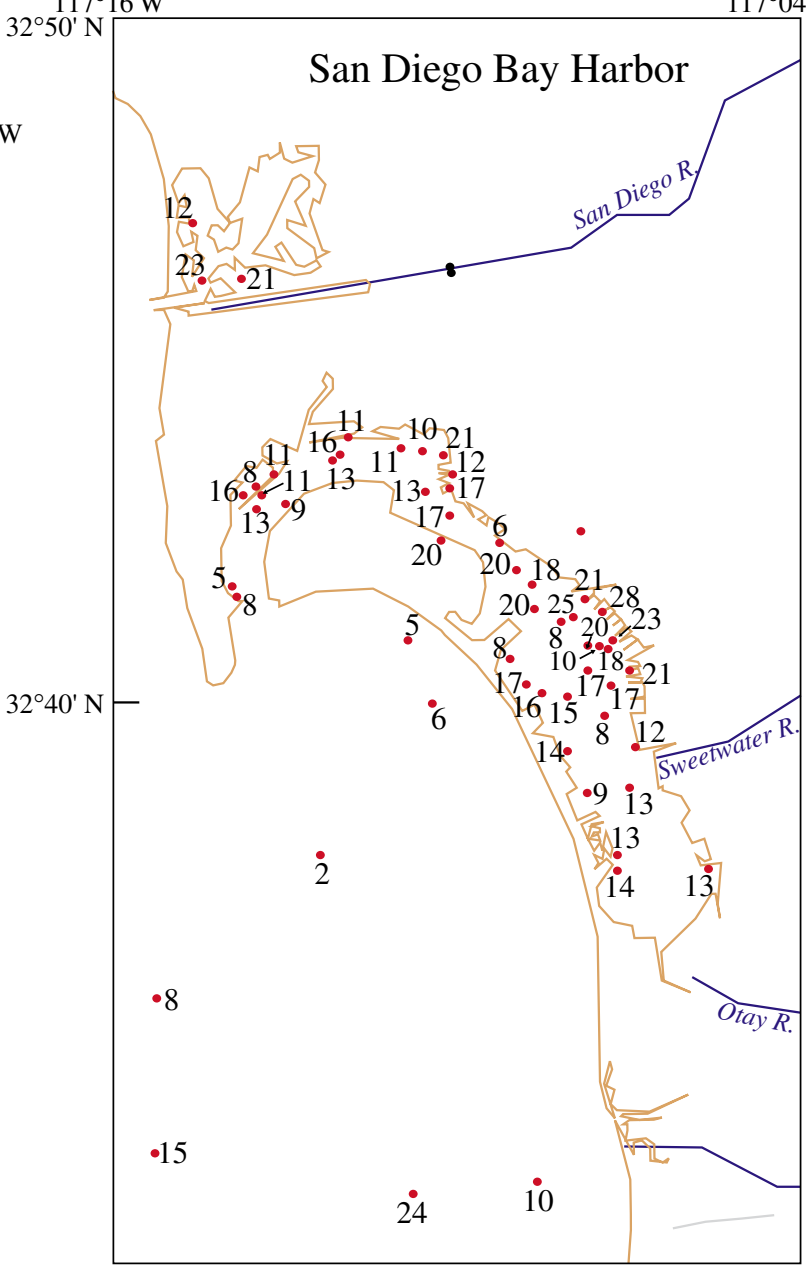

Figure 7. Smectite content of the $<2 \mu \mathrm{m}$ size-fraction of harbor sediments 


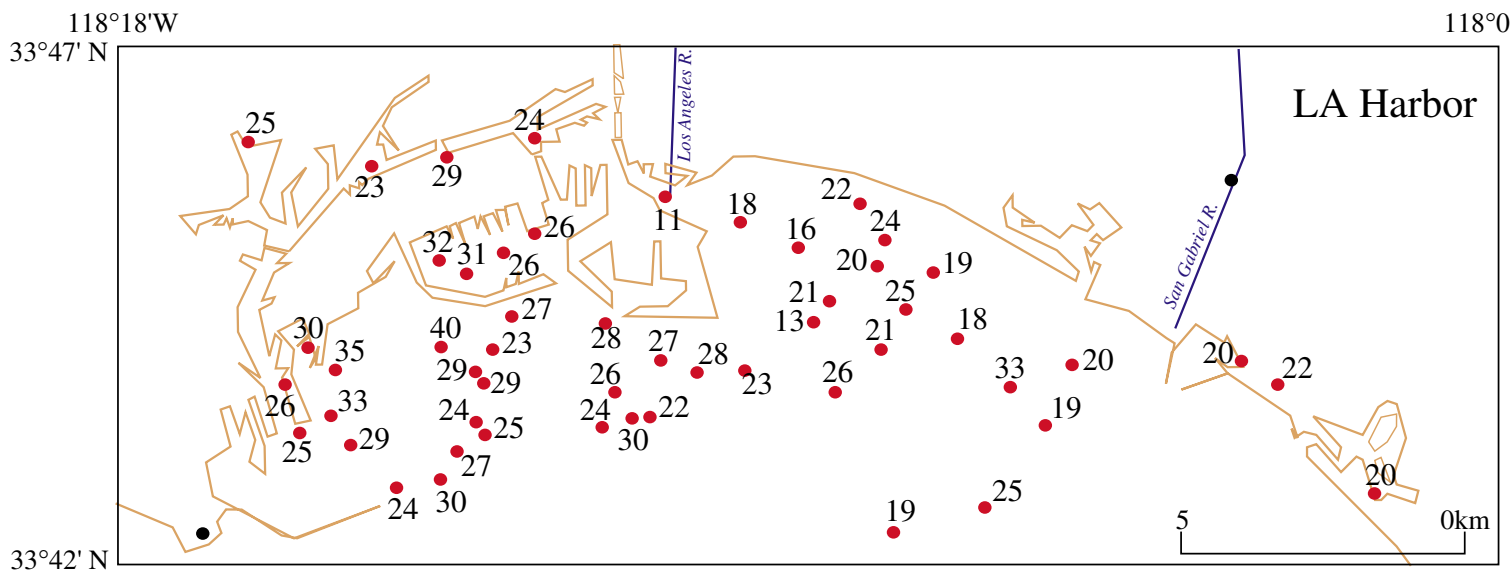

$117^{\circ} 58^{\prime} \mathrm{W}$

$33^{\circ} 40^{\prime} \mathrm{N}$

$33^{\circ} 40^{\prime}$

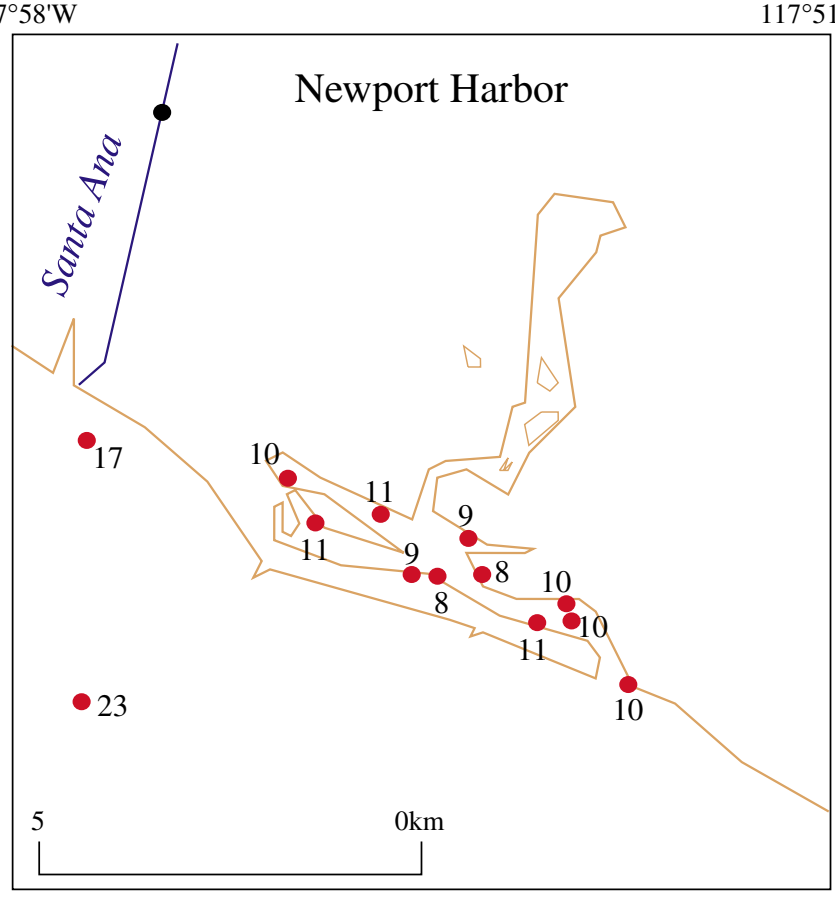

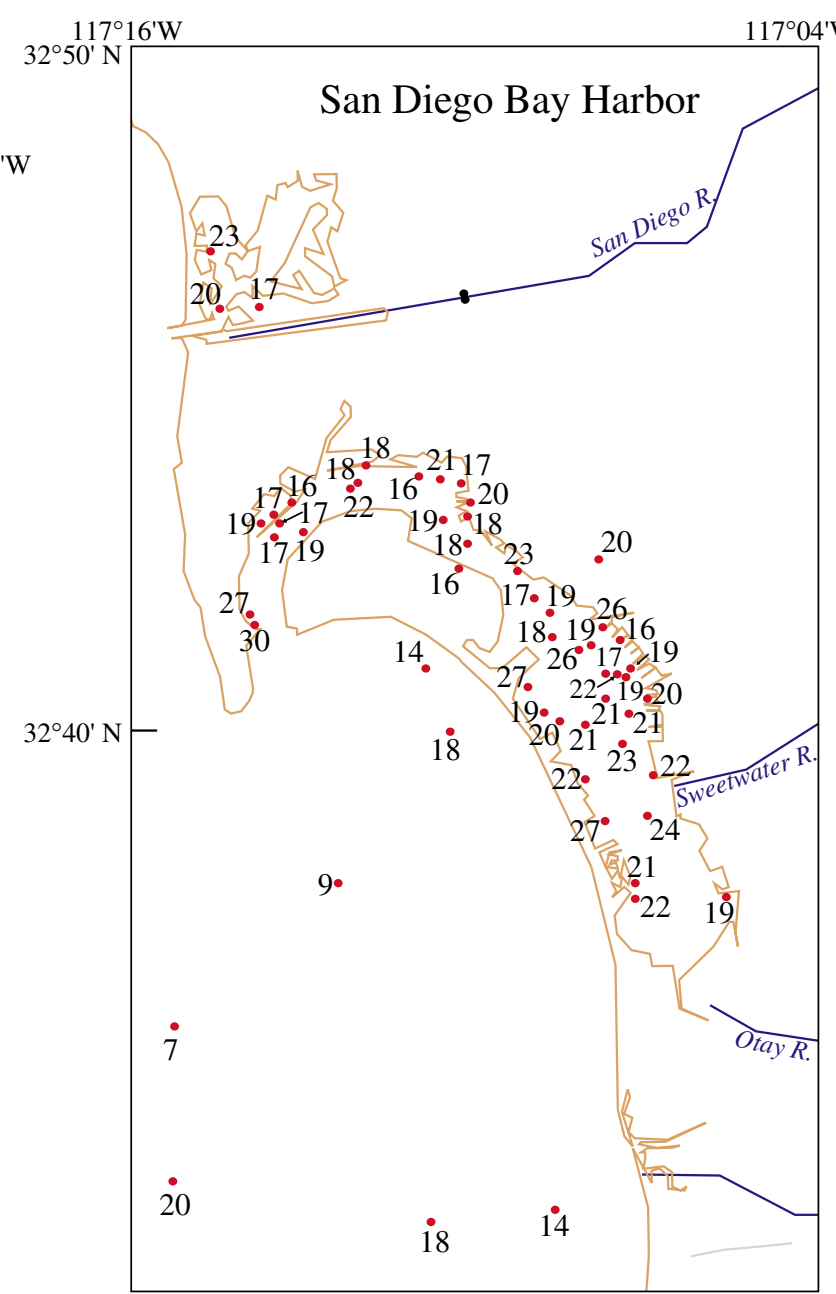

Figure 8. Kaolinite + chlorite content of the $<2 \mu \mathrm{m}$ size-fraction of harbor sediments 


\section{\% EXPANDABLE LAYERS}

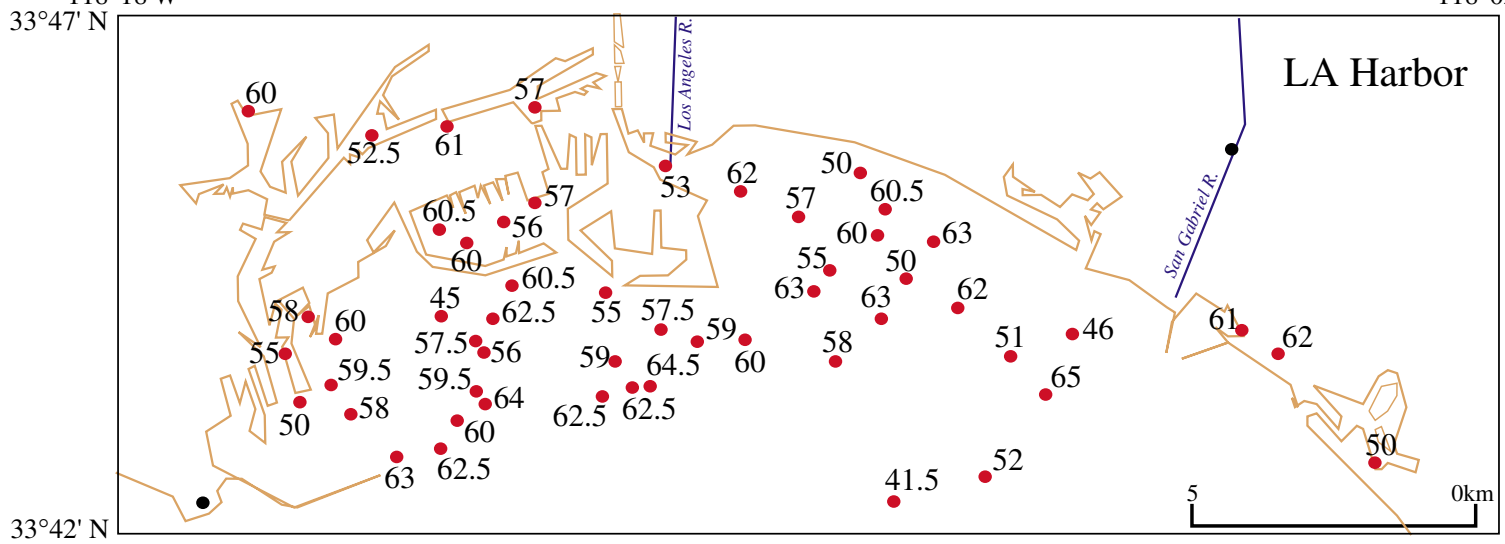

$33^{\circ} 40^{\prime} \mathrm{N}$

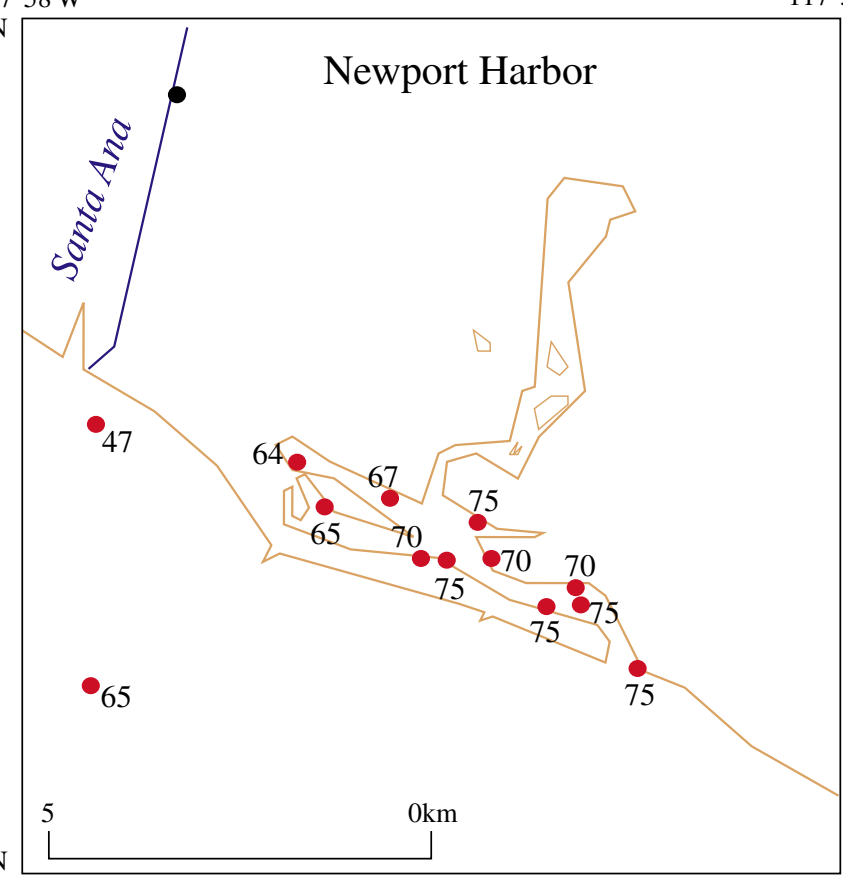

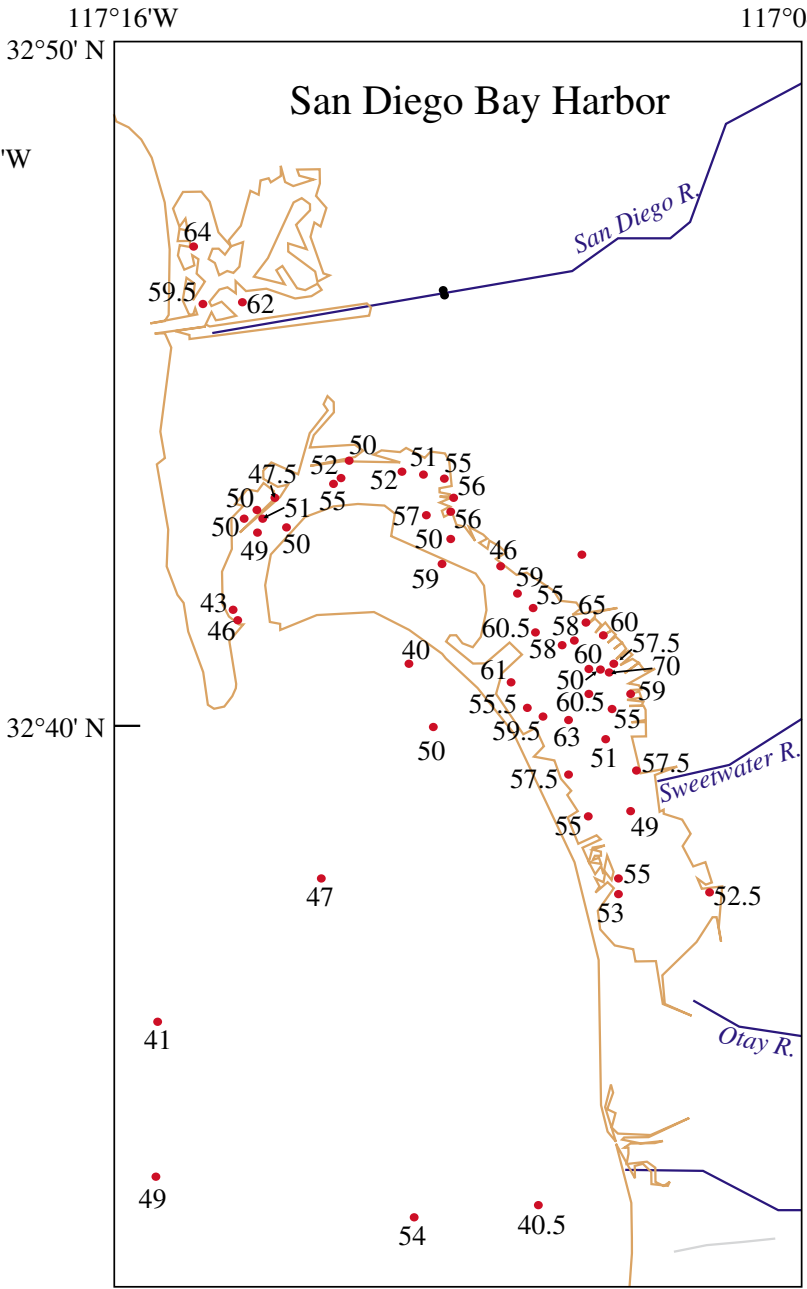

Figure 9. Percent expandable layers in the smectite phase of the $<2 \mu \mathrm{m}$ size-fraction of harbor sediments 